\title{
Robust Adaptive Neural Backstepping Control for a Class of Nonlinear Systems with Dynamic Uncertainties
}

\author{
Hongyan Yang, ${ }^{1}$ Huanqing Wang, ${ }^{1}$ and Hamid Reza Karimi ${ }^{2}$ \\ ${ }^{1}$ School of Mathematics and Physics, Bohai University, Jinzhou, Liaoning 121000, China \\ ${ }^{2}$ Department of Engineering, Faculty of Engineering and Science, University of Agder, 4898 Grimstad, Norway \\ Correspondence should be addressed to Huanqing Wang; ndwhq@163.com
}

Received 15 April 2014; Accepted 9 June 2014; Published 2 July 2014

Academic Editor: Josep M. Rossell

Copyright (c) 2014 Hongyan Yang et al. This is an open access article distributed under the Creative Commons Attribution License, which permits unrestricted use, distribution, and reproduction in any medium, provided the original work is properly cited.

\begin{abstract}
This paper is concerned with adaptive neural control of nonlinear strict-feedback systems with nonlinear uncertainties, unmodeled dynamics, and dynamic disturbances. To overcome the difficulty from the unmodeled dynamics, a dynamic signal is introduced. Radical basis function (RBF) neural networks are employed to model the packaged unknown nonlinearities, and then an adaptive neural control approach is developed by using backstepping technique. The proposed controller guarantees semiglobal boundedness of all the signals in the closed-loop systems. A simulation example is given to show the effectiveness of the presented control scheme.
\end{abstract}

\section{Introduction}

In the past decades, much attention has been paid on the control design of complex nonlinear systems [1-11]. Many remarkable control approaches in this area have been developed, including adaptive backstepping technique [1-3], fault tolerant control [12-17], and and fuzzy control [18-29]. In particular, adaptive backstepping approach has played an important role in the control of strict-feedback nonlinear systems. Generally, adaptive backstepping provides a systematic control approach to solve the tracking or regulation control problems of uncertain nonlinear systems, in which the classic adaptive control is applied to deal with the unknown parameter and backstepping technique is used to construct controller. The main feature of adaptive backstepping control is that it can handle the control problems of nonlinear systems without the requirement of matching condition. Adaptive backstepping technique was provided in [1] to obtain global stability and asymptotic tracking performance for parametric strict-feedback systems with overparameterization, and the overparameterization was overcome by applying the tuning functions in [2]. Then, a backstepping-based design was extensively utilized to control different types of nonlinear systems [30-35]. All the above control methods, however, assume that the nonlinear functions of the control systems are either known or bounded by known functions multiplying uncertain parameters. This restriction makes the aforementioned methods inapplicable to the control of the systems with unknown continuous nonlinear functions.

On the other hand, approximation-based adaptive neural (or fuzzy) backstepping control has received increasing attention in recent years. In general, approximation-based adaptive backstepping technique is an effective control approach for handling the control problem of highly uncertain complex nonlinear strict-feedback systems, in which neural networks or fuzzy systems are utilized to model the unknown nonlinear functions. So far, there exist some elegant results; see, for example, [36-54] and the references therein. By applying adaptive neural control together with backstepping, in [36-43], many control approaches are developed for single-input and single-output (SISO) nonlinear systems or multi-input and multioutput (MIMO) nonlinear systems. Alternatively, several fuzzy adaptive control strategies [19, 44-55] were developed to deal with the control problem of uncertain nonlinear systems with strict-feedback form. However, the above adaptive neural or fuzzy backstepping control approaches required the controlled strict-feedback nonlinear systems to be free of the unmodeled dynamics and 
dynamic disturbances. As stated in $[56,57]$, the unmodeled dynamics and dynamic disturbances often appear in practical systems $[58,59]$ due to the measurement noise, modeling errors, external disturbances, modeling simplifications, or changes with time variations, and they are also the resources of the instability of the considered systems. Therefore, some researchers have concentrated on the problem of control design for nonlinear systems with unmodeled dynamics and dynamic disturbances. In $[56,57]$, the problem of adaptive backstepping control was investigated for a class of nonlinear systems with dynamics uncertainties, in which the nonlinear functions were assumed to be linear combinations of the known functions with unknown parameters. Furthermore, by using the approximation properties of fuzzy logic systems, Tong et al. $[58,59]$ developed several fuzzy adaptive control approaches for nonlinear systems in strict-feedback form, where the number of adaptation laws depends on the number of fuzzy base functions. The more fuzzy rules are applied to improve approximation accuracy, the more adaptive parameters will be needed, and, in this way, the online learning time may be very large.

Inspired by previous works, this paper focuses on the problem of adaptive neural control for nonlinear strictfeedback systems with unmodeled dynamics and dynamic disturbances. During the controller design, a dynamic signal is introduced to handle the unmodeled dynamics and RBF neural networks are used to approximate the unknown nonlinearities, and then an adaptive neural control scheme is systematically derived via backstepping. The proposed controller guarantees that all the signals in the closedloop systems are semiglobally uniformly ultimately bounded (SGUUB) in the sense of mean square. Compared with the control approaches $[58,59]$, the main contributions of this paper are summarized as follows: (1) the strict limitation to the dynamic disturbances is relaxed, which can refer to Remark 3; (2) by estimating the norm of the weight vector of neural networks basis functions, the number of adaptive parameters is not more than the order of the considered nonlinear system. As a result, the burdensome computation is significantly alleviated, which makes our control design more suitable for the practical applications.

The remainder of the paper is organized as follows. Section 2 begins with the problem formulation and some preliminaries. A backstepping-based adaptive control scheme is design in Section 3. In Section 4, a numerical example is given. Finally, the conclusion of this paper is shown in Section 5 .

\section{Problem Formulation and Preliminaries}

In this paper, we consider a class of nonlinear strict-feedback systems described by

$$
\begin{aligned}
\dot{z} & =q(z, x) \\
\dot{x}_{i} & =g_{i}\left(\bar{x}_{i}\right) x_{i+1}+f_{i}\left(\bar{x}_{i}\right)+\Delta_{i}(x, z, t), \quad i=1, \ldots, n-1, \\
\dot{x}_{n} & =g_{n}\left(\bar{x}_{n}\right) u+f_{n}\left(\bar{x}_{n}\right)+\Delta_{n}(x, z, t),
\end{aligned}
$$

where $x=\left[x_{1}, x_{2}, \ldots, x_{n}\right]^{T} \in R^{n}, u \in R$, and $y \in R$ are the system state, control input, and system output, respectively, $\bar{x}_{i}=\left[x_{1}, x_{2}, \ldots, x_{i}\right]^{T} \in R^{i}, f_{i}(\cdot)$, and $g_{i}(\cdot)$ are unknown smooth functions, and $\Delta_{i}(i=1,2, \ldots, n)$ are nonlinear dynamic disturbances. The $z$-dynamics in (1) denotes the unmodeled dynamics.

Remark 1. It is worth noting that many practical systems such as the electromechanical system [59] transformable into (1) have been investigated extensively during the last decades from both theoretical and practical viewpoints; see, for example, [56-59].

In order to facilitate the control design later, the below assumptions are imposed on the system (1).

Assumption 2. For the dynamic disturbances $\Delta_{i}(i=$ $1,2, \ldots, n)$ in $(1)$, there exist unknown nonnegative smooth functions $\phi_{i 1}(\cdot)$ and $\phi_{i 2}(\cdot)$, such that

$$
\left|\Delta_{i}(x, z, t)\right| \leq \phi_{i 1}\left(\left|\left(x_{1}, \ldots, x_{i}\right)\right|\right)+\phi_{i 2}(|z|) .
$$

Remark 3. This assumption is similar to the one in $[58,59]$ in which $\phi_{i 1}(\cdot)$ and $\phi_{i 2}(\cdot)$ are known. Assumption 2, however, does not require them to be known. Therefore, Assumption 2 relaxes the restriction in the existing results.

Assumption 4 (see [59]). The unmodeled dynamics in (1) is exponentially input-to-state practically stable (exp-ISpS); that is, for the system $\dot{z}=q(z, x)$, there exists an exp-ISpS Lyapunov function $V(z)$ such that

$$
\begin{gathered}
\alpha_{1}(|z|) \leq V(z) \leq \alpha_{2}(|z|), \\
\frac{\partial V(z)}{\partial z} q(z, x) \leq-c_{0} V(z)+\mu(|x|)+d_{0},
\end{gathered}
$$

where $\alpha_{1}, \alpha_{2}$, and $\mu$ are of class $K_{\infty}$-functions and $c_{0}$ and $d_{0}$ are known positive constants.

Assumption 5 (see [50]). For $1 \leq i \leq n$, the signs of $g_{i}\left(\bar{x}_{i}\right)$ are known, and there exists unknown positive constant $b$ such that

$$
0<b \leq\left|g_{i}\left(\bar{x}_{i}\right)\right|<\infty, \quad \forall \bar{x}_{i} \in R^{i} .
$$

Remark 6. Equation (4) implies that $g_{i}\left(\bar{x}_{i}\right)$ are either strictly positive or negative. Without loss of generality, it is supposed that $0<b \leq g_{i}\left(\bar{x}_{i}\right)$. In addition, since $b$ is not required in the designed controller, its true value is not required to be known.

Lemma 7 (see [59]). If $V$ is an exp-ISpS Lyapunov function for a control system, that is, (3) hold, then, for any constants $\bar{c}$ in $\left(0, c_{0}\right)$, any initial condition $x_{0}=x_{0}(0)$, and any function $\bar{\mu}\left(x_{1}\right) \geq \mu\left(\left|x_{1}\right|\right)$, there exists a finite time $T_{0}=T_{0}\left(\bar{c}, r_{0}, z_{0}\right)$, a nonnegative function $D(t)$ defined for all $t \geq 0$, and a signal described by

$$
\dot{r}=-\bar{c} r+\bar{\mu}\left(x_{1}(t)\right)+d_{0}, \quad r(0)=r_{0},
$$

such that $D(t)=0$ for all $t \geq T_{0}$,

$$
V(z(t)) \leq r(t)+D(t) .
$$


For all $t \geq 0$, the solutions are defined. Without loss of generality, this paper takes $\bar{\mu}(\cdot)$ as $\bar{\mu}(s)=s^{2} \mu_{0}\left(s^{2}\right)$, where $\bar{\mu}(\cdot)$ is a nonnegative smooth function. Therefore, the dynamical $r$ defined by (5) becomes

$$
\dot{r}=-\bar{c} r+x_{1}^{2} \mu_{0}\left(\left|x_{1}^{2}\right|\right)+d_{0}, \quad r(0)=r_{0},
$$

where $\mu_{0}$ is a nonnegative smooth function.

Throughout this paper, RBF neural networks are applied to model the unknown continuous nonlinear functions. In [60], it has been indicated that, with enough node number $l$, the RBF neural networks $\phi^{* T} \xi(X)$ can model the continuous function $f(X)$ within a compact set $\Omega_{X} \subset R^{q}$ to arbitrary accuracy $\varepsilon>0$ as

$$
f(X)=\phi^{* T} \xi(X)+\delta(X), \quad \forall X \in \Omega_{X} \in R^{q},
$$

in which $\phi^{*}$ denotes the ideal weight vector and is specified as

$$
\phi^{*}:=\arg \min _{\phi \in \bar{R}^{N}}\left\{\sup _{X \in \Omega_{X}}\left|f(X)-\phi^{T} \xi(X)\right|\right\}
$$

$\delta(X)$ depicts the approximation error satisfying $|\delta(X)| \leq \varepsilon$, $\phi^{*}=\left[\phi_{1}, \phi_{2}, \ldots, \phi_{l}\right]^{T} \in R^{l}$ is the weight vector, and $\xi(X)=$ $\left[\xi_{1}(X), \xi_{2}(X), \ldots, \xi_{l}(X)\right]^{T}$ is the basis function vector with $l$ being the number of the neural networks nodes and $l>1$. The basis function $\xi_{i}(X)$ is taken as the Gaussian function in the below form:

$$
\xi_{i}(X)=\exp \left[-\frac{\left(X-\mu_{i}\right)^{T}\left(X-\mu_{i}\right)}{\eta_{i}^{2}}\right], \quad(i=1,2, \ldots, l),
$$

where $\mu_{i}=\left[\mu_{i 1}, \mu_{i 2}, \ldots, \mu_{i q}\right]^{T}$ and $\eta_{i}$ are the center of the receptive field and the width of the Gaussian function, respectively.

\section{Adaptive Neural Control Design}

In this section, the adaptive backstepping control design for system (1) is proposed. As usual, in the backstepping approach, the following coordinate transformation is made:

$$
z_{i}=x_{i}-\alpha_{i-1}, \quad i=1,2, \ldots, n,
$$

where $\alpha_{0}=0, \alpha_{i}$ is the virtual control signal and will be constructed at Step $i$, and the actual controller $u$ will be designed at Step $n$. Now, we begin the controller design procedure.

Step 1. Consider the following subsystem:

$$
\begin{gathered}
\dot{z}=q(z, x), \\
\dot{x}_{1}=g_{1}\left(x_{1}\right) x_{2}+f_{1}\left(x_{1}\right)+\Delta_{1}(x, z, t) .
\end{gathered}
$$

Based on $z_{1}=x_{1}$, then choose Lyapunov functions as

$$
V_{1}=\frac{1}{2} z_{1}^{2}+\frac{1}{\lambda_{0}} r+\frac{b}{2 \gamma_{1}} \widetilde{\theta}_{1}^{2}
$$

where $\bar{\mu}\left(x_{1}\right)=x_{1}^{2} \mu_{0}\left(x_{1}^{2}\right), \lambda_{0}$ and $\gamma_{1}$ are positive design parameters, and $\widetilde{\theta}_{1}=\theta_{1}-\widehat{\theta}_{1}$ is the parameter error with $\widehat{\theta}_{1}$ being the estimation of $\theta_{1}$ which is defined later.

By taking (12) into account, we have

$$
\begin{aligned}
\dot{V}_{1} & =z_{1}\left(g_{1}\left(x_{1}\right) x_{2}+f_{1}\left(x_{1}\right)+\Delta_{1}\right)+\frac{1}{\lambda_{0}} \dot{r}-\frac{b}{\gamma_{1}} \widetilde{\theta}_{1} \dot{\hat{\theta}}_{1} \\
& \leq z_{1}\left(g_{1}\left(x_{1}\right) x_{2}+f_{1}\left(x_{1}\right)\right)+\left|z_{1}\right|\left|\Delta_{1}\right|+\frac{1}{\lambda_{0}} \dot{r}-\frac{b}{\gamma_{1}} \widetilde{\theta}_{1} \dot{\hat{\theta}}_{1} .
\end{aligned}
$$

By Assumption 2, it follows that

$$
\begin{aligned}
\dot{V}_{1} & \leq z_{1}\left(g_{1}\left(x_{1}\right) x_{2}+f_{1}\left(x_{1}\right)\right)+\left|z_{1}\right| \phi_{11}\left(\left|x_{1}\right|\right) \\
& +\left|z_{1}\right| \phi_{12}(|z|)+\frac{1}{\lambda_{0}}\left(x_{1}^{2} \mu_{0}\left(x_{1}^{2}\right)+d_{0}\right)-\frac{\bar{c}}{\lambda_{0}} r-\frac{b}{\gamma_{1}} \widetilde{\theta}_{1} \dot{\hat{\theta}}_{1} .
\end{aligned}
$$

Then, we will deal with the third and fourth terms in (15), respectively. By using $0 \leq|x|-x \tanh (x / \epsilon) \leq 0.2785 \epsilon=\epsilon^{\prime}$, for $\forall \epsilon>0$, we have

$$
\begin{aligned}
\left|z_{1}\right| \phi_{11}\left(\left|x_{1}\right|\right) & \\
= & \left|z_{1} \phi_{11}\left(\left|x_{1}\right|\right)\right|-z_{1} \phi_{11}\left(\left|x_{1}\right|\right) \tanh \left(\frac{z_{1} \phi_{11}\left(\left|x_{1}\right|\right)}{\epsilon_{11}}\right) \\
& +z_{1} \phi_{11}\left(\left|x_{1}\right|\right) \tanh \left(\frac{z_{1} \phi_{11}\left(\left|x_{1}\right|\right)}{\epsilon_{11}}\right) \\
\leq & \epsilon_{11}^{\prime}+z_{1} \phi_{11}\left(\left|x_{1}\right|\right) \tanh \left(\frac{z_{1} \phi_{11}\left(\left|x_{1}\right|\right)}{\epsilon_{11}}\right) \\
\leq & z_{1} \widehat{\phi}_{11}\left(x_{1}\right)+\epsilon_{11}^{\prime},
\end{aligned}
$$

where $\epsilon_{11}^{\prime}=0.2785 \epsilon_{11}$ and $\widehat{\phi}_{11}\left(x_{1}\right)=$ $\phi_{11}\left(\left|x_{1}\right|\right) \tanh \left(z_{1} \phi_{11}\left(\left|x_{1}\right|\right) / \epsilon_{11}\right)$ is a smooth function.

By using the same derivations as [58], one has

$$
\begin{aligned}
\left|z_{1}\right| \phi_{12}(|z|) \leq & \left|z_{1}\right| \bar{\phi}_{12}(r)+\frac{1}{4} z_{1}^{2}+d_{1}(t) \\
\leq & z_{1} \bar{\phi}_{12}(r) \tanh \left(\frac{z_{1} \bar{\phi}_{12}(r)}{\epsilon_{12}}\right)+\epsilon_{12}^{\prime} \\
& +\frac{1}{4} z_{1}^{2}+d_{1}(t) \\
\leq & z_{1} \widehat{\phi}_{12}\left(x_{1}, r\right)+\epsilon_{12}^{\prime}+\frac{1}{4} z_{1}^{2}+d_{1}(t),
\end{aligned}
$$

where $\epsilon_{12}^{\prime}=0.2785 \epsilon_{12}, \bar{\phi}_{12}(r)=\phi_{12} \circ \alpha_{1}^{-1}(2 r), d_{1}(t)=\left(\phi_{12} \circ\right.$ $\left.\alpha_{1}^{-1}(2 D(t))\right)^{2}$, and $\widehat{\phi}_{12}\left(x_{1}, r\right)=\bar{\phi}_{12}(r) \tanh \left(z_{1} \bar{\phi}_{12}(r) / \epsilon_{12}\right)$.

Subsequently, substituting (16) and (17) into (15) gives

$$
\begin{array}{r}
\dot{V}_{1} \leq z_{1}\left(g_{1}\left(x_{1}\right) z_{2}+g_{1}\left(x_{1}\right) \alpha_{1}+\widehat{f}_{1}\left(Z_{1}\right)\right)-\frac{z_{1}^{2}}{2} \\
+\frac{d_{0}}{\lambda_{0}}-\frac{\bar{c}}{\lambda_{0}} r-\frac{b}{\gamma_{1}} \tilde{\theta}_{1} \dot{\hat{\theta}}_{1}+\epsilon_{11}^{\prime}+\epsilon_{12}^{\prime}+d_{1}(t),
\end{array}
$$


where the function $\widehat{f}_{1}\left(Z_{1}\right)$ is defined as

$$
\begin{aligned}
\widehat{f}_{1}\left(Z_{1}\right)= & f_{1}\left(x_{1}\right)+\widehat{\phi}_{11}\left(x_{1}\right)+\widehat{\phi}_{12}(r)+\frac{3}{4} z_{1} \\
& +\frac{1}{\lambda_{0}}\left(x_{1} \mu_{0}\left(x_{1}^{2}\right)\right),
\end{aligned}
$$

where $Z_{1}=\left[x_{1}, r\right]^{T} \in \Omega_{Z_{1}} \subset R^{2}$.

Since the smooth function $\hat{f}_{1}\left(Z_{1}\right)$ is unknown, it cannot be implemented in practice. By employing RBF neural network in $\phi_{1}^{T} \xi\left(Z_{1}\right)$ to approximate $\widehat{f}_{1}\left(Z_{1}\right)$, we have

$$
\widehat{f}_{1}\left(Z_{1}\right)=\phi_{1}^{T} \xi_{1}\left(Z_{1}\right)+\delta_{1}\left(Z_{1}\right), \quad\left|\delta_{1}\left(Z_{1}\right)\right| \leq \epsilon_{13},
$$

where $\delta_{1}\left(Z_{1}\right)$ denotes an approximation error and $\epsilon_{13}>0$ is a given positive constant.

Next, the following result can be obtained by substituting (20) into (18):

$$
\begin{gathered}
\dot{V}_{1} \leq g_{1}\left(x_{1}\right) z_{1} z_{2}+g_{1}\left(x_{1}\right) z_{1} \alpha_{1}+z_{1} \phi_{1}^{T} \xi_{1}\left(Z_{1}\right)+z_{1} \delta_{1}\left(Z_{1}\right) \\
-\frac{z_{1}^{2}}{2}+\frac{d_{0}}{\lambda_{0}}-\frac{\bar{c}}{\lambda_{0}} r-\frac{b}{\gamma_{1}} \tilde{\theta}_{1} \dot{\hat{\theta}}_{1}+\epsilon_{11}^{\prime}+\epsilon_{12}^{\prime}+d_{1}(t) .
\end{gathered}
$$

By using

$$
\begin{aligned}
z_{1} \phi_{1}^{T} \xi_{1}\left(Z_{1}\right) & \leq \frac{b \theta_{1}}{2 \eta_{1}^{2}} \xi_{1}^{T}\left(Z_{1}\right) \xi_{1}\left(Z_{1}\right) z_{1}^{2}+\frac{\eta_{1}^{2}}{2}, \\
z_{1} \delta_{1}\left(Z_{1}\right) & \leq \frac{z_{1}^{2}}{2}+\frac{\epsilon_{13}^{2}}{2},
\end{aligned}
$$

one has

$$
\begin{aligned}
\dot{V}_{1} \leq & g_{1}\left(x_{1}\right) z_{1} z_{2}+g_{1}\left(x_{1}\right) z_{1} \alpha_{1}+\frac{b \theta_{1}}{2 \eta_{1}^{2}} \xi_{1}^{T}\left(Z_{1}\right) \xi_{1}\left(Z_{1}\right) z_{1}^{2} \\
& +\frac{\eta_{1}^{2}}{2}+\frac{\epsilon_{13}^{2}}{2}+\frac{d_{0}}{\lambda_{0}}-\frac{\bar{c}}{\lambda_{0}} r-\frac{b}{\gamma_{1}} \tilde{\theta}_{1} \dot{\hat{\theta}}_{1}+\epsilon_{11}^{\prime}+\epsilon_{12}^{\prime}+d_{1}(t)
\end{aligned}
$$

with $\theta_{1}=b^{-1}\left\|\phi_{1}\right\|^{2}$ being an unknown parameter.

Construct the virtual control signal $\alpha_{1}$ as

$$
\alpha_{1}=-k_{1} z_{1}-\frac{\widehat{\theta}_{1}}{2 \eta_{1}^{2}} z_{1} \xi_{1}^{T}\left(Z_{1}\right) \xi_{1}\left(Z_{1}\right),
$$

where $k_{1}$ and $\eta_{1}$ are positive design constants.

By taking Assumption 5 into account, one has

$$
g_{1}\left(x_{1}\right) z_{1} \alpha_{1} \leq-k_{1} b z_{1}^{2}-\frac{\widehat{\theta}_{1}}{2 \eta_{1}^{2}} b z_{1}^{2} \xi_{1}^{T}\left(Z_{1}\right) \xi_{1}\left(Z_{1}\right) .
$$

Further, by substituting (25) into (23), we obtain

$$
\begin{aligned}
\dot{V}_{1} \leq & -b k_{1} z_{1}^{2}-\frac{\bar{c}}{\lambda_{0}} r+\frac{b \widetilde{\theta}_{1}}{\gamma_{1}}\left(\frac{\gamma_{1}}{2 \eta_{1}^{2}} \xi_{1}^{T}\left(Z_{1}\right) \xi_{1}\left(Z_{1}\right) z_{1}^{2}-\dot{\hat{\theta}}_{1}\right) \\
& +g_{1}\left(x_{1}\right) z_{1} z_{2}+\frac{\eta_{1}^{2}}{2}+\frac{\epsilon_{13}^{2}}{2}+\frac{d_{0}}{\lambda_{0}}+\epsilon_{11}^{\prime}+\epsilon_{12}^{\prime}+d_{1}(t) .
\end{aligned}
$$

Next, we choose the adaptive law in the following form:

$$
\dot{\hat{\theta}}_{1}=\frac{\gamma_{1}}{2 \eta_{1}^{2}} \xi_{1}^{T}\left(Z_{1}\right) \xi_{1}\left(Z_{1}\right) z_{1}^{2}-\sigma_{1} \widehat{\theta}_{1}
$$

where $\eta_{1}$ and $\sigma_{1}$ are design parameters.

By using (27), we can rewrite (26) as

$$
\begin{aligned}
\dot{V}_{1} \leq & -b k_{1} z_{1}^{2}-\frac{\bar{c}}{\lambda_{0}} r+g_{1}\left(x_{1}\right) z_{1} z_{2}+\frac{b \sigma_{1} \widetilde{\theta}_{1} \widehat{\theta}_{1}}{\gamma_{1}} \\
& +\frac{\eta_{1}^{2}}{2}+\frac{\epsilon_{13}^{2}}{2}+\frac{d_{0}}{\lambda_{0}}+\epsilon_{11}^{\prime}+\epsilon_{12}^{\prime}+d_{1}(t) .
\end{aligned}
$$

Noting

$$
\frac{b \sigma_{1} \widetilde{\theta}_{1} \widehat{\theta}_{1}}{\gamma_{1}}=-\frac{b \sigma_{1} \widetilde{\theta}_{1}^{2}}{\gamma_{1}}+\frac{b \sigma_{1} \widetilde{\theta}_{1} \theta_{1}}{\gamma_{1}} \leq-\frac{b \sigma_{1} \widetilde{\theta}_{1}^{2}}{2 \gamma_{1}}+\frac{b \sigma_{1} \theta_{1}^{2}}{2 \gamma_{1}}
$$

then the following inequality holds:

$$
\dot{V}_{1} \leq-b k_{1} z_{1}^{2}-\frac{\bar{c}}{\lambda_{0}} r-\frac{b \sigma_{1} \widetilde{\theta}_{1}^{2}}{2 \gamma_{1}}+\frac{d_{0}}{\lambda_{0}}+D_{1}+g_{1}\left(x_{1}\right) z_{1} z_{2}
$$

where $D_{1}=\eta_{1}^{2} / 2+b \sigma_{1} \theta_{1}^{2} / 2 \gamma_{1}+\epsilon_{13}^{2} / 2+\epsilon_{11}^{\prime}+\epsilon_{12}^{\prime}+d_{1}(t)$.

Step 2. Based on $z_{2}=x_{2}-\alpha_{1}$, then the time derivative of $z_{2}$ is given by

$$
\begin{aligned}
\dot{z}_{2}= & \dot{x}_{2}-\dot{\alpha}_{1} \\
= & g_{2}\left(\bar{x}_{2}\right) x_{3}+f_{2}\left(\bar{x}_{2}\right)+\Delta_{2} \\
& -\frac{\partial \alpha_{1}}{\partial x_{1}}\left(g_{1}\left(x_{1}\right) x_{2}+f_{1}\left(x_{1}\right)+\Delta_{1}\right)-\frac{\partial \alpha_{1}}{\partial \hat{\theta}_{1}} \dot{\hat{\theta}}_{1}-\frac{\partial \alpha_{1}}{\partial r} \dot{r} \\
= & g_{2}\left(\bar{x}_{2}\right) x_{3}+\left(f_{2}\left(\bar{x}_{2}\right)-\frac{\partial \alpha_{1}}{\partial x_{1}} f_{1}\left(x_{1}\right)\right)+\bar{\Delta}_{2} \\
& -\frac{\partial \alpha_{1}}{\partial x_{1}} g_{1}\left(x_{1}\right) x_{2}-\frac{\partial \alpha_{1}}{\partial \hat{\theta}_{1}} \dot{\hat{\theta}}_{1}-\frac{\partial \alpha_{1}}{\partial r} \dot{r},
\end{aligned}
$$

where $\bar{\Delta}_{2}=\Delta_{2}-\left(\partial \alpha_{1} / \partial x_{1}\right) \Delta_{1}$.

Construct the Lyapunov function

$$
V_{2}=V_{1}+\frac{1}{2} z_{2}^{2}+\frac{b}{2 \gamma_{2}} \widetilde{\theta}_{2}^{2}
$$


The derivative of $V_{2}$ is

$$
\begin{aligned}
\dot{V}_{2} \leq & -b k_{1} z_{1}^{2}-\frac{b \sigma_{1} \widetilde{\theta}_{1}^{2}}{2 \gamma_{1}}-\frac{\bar{c}}{\lambda_{0}} r \\
+ & z_{2}\left[g_{2}\left(\bar{x}_{2}\right) x_{3}+g_{1}\left(x_{1}\right) z_{1}+f_{2}\left(\bar{x}_{2}\right)-\frac{\partial \alpha_{1}}{\partial x_{1}} f_{1}\left(x_{1}\right)\right. \\
& \left.-\frac{\partial \alpha_{1}}{\partial x_{1}} g_{1}\left(x_{1}\right) x_{2}-\frac{\partial \alpha_{1}}{\partial \widehat{\theta}_{1}}-\frac{\partial \alpha_{1}}{\partial r} \dot{r}\right] \\
+ & \left|z_{2} \bar{\Delta}_{2}\right|+D_{1}+\frac{d_{0}}{\lambda_{0}}-\frac{b}{\gamma_{2}} \widetilde{\theta}_{2} \dot{\hat{\theta}}_{2} .
\end{aligned}
$$

By Assumption 2, we have

$$
\begin{aligned}
\left|z_{2} \bar{\Delta}_{2}\right|= & \left|z_{2}\right|\left|\Delta_{2}-\frac{\partial \alpha_{1}}{\partial x_{1}} \Delta_{1}\right| \\
\leq & \left|z_{2}\right|\left(\left|\Delta_{2}\right|+\left|\frac{\partial \alpha_{1}}{\partial x_{1}}\right|\left|\Delta_{1}\right|\right) \\
\leq & \left|z_{2}\right|\left(\phi_{21}+\left|\frac{\partial \alpha_{1}}{\partial x_{1}}\right| \phi_{11}\right) \\
& +\left|z_{2}\right|\left(\phi_{22}(|z|)+\left|\frac{\partial \alpha_{1}}{\partial x_{1}}\right| \phi_{12}(|z|)\right) .
\end{aligned}
$$

Similar to the estimation methods in (35), the following results can be obtained:

$$
\begin{gathered}
\left|z_{2}\right|\left(\phi_{21}+\left|\frac{\partial \alpha_{1}}{\partial x_{1}}\right| \phi_{11}\right) \leq z_{2} \widehat{\phi}_{21}\left(\bar{x}_{2}, \widehat{\theta}_{1}, r\right)+\epsilon_{21}^{\prime}, \\
\left|z_{2}\right|\left(\phi_{22}(|z|)+\left|\frac{\partial \alpha_{1}}{\partial x_{1}}\right| \phi_{12}(|z|)\right) \\
\leq\left|z_{2}\right|\left(\phi_{22} \circ \alpha_{1}^{-1}(2 r)+\left|\frac{\partial \alpha_{1}}{\partial x_{1}}\right| \phi_{12} \circ \alpha_{1}^{-1}(2 r)\right) \\
+\left|z_{2}\right| \phi_{22} \circ \alpha_{1}^{-1}(2 D(t)) \\
+\left|z_{2}\right|\left|\frac{\partial \alpha_{1}}{\partial x_{1}}\right| \phi_{12} \circ \alpha_{1}^{-1}(2 D(t)) \\
\leq\left|z_{2}\right| \bar{\phi}_{22}\left(x_{1}, \widehat{\theta}_{1}, r\right)+\frac{z_{2}^{2}}{4}\left(1+\left(\frac{\partial \alpha_{1}}{\partial x_{1}}\right)^{2}\right)+d_{2}(t) \\
\leq \\
z_{2} \widehat{\phi}_{22}\left(x_{1}, \widehat{\theta}_{1}, r\right)+\epsilon_{22}^{\prime}+\frac{z_{2}^{2}}{4}\left(1+\left(\frac{\partial \alpha_{1}}{\partial x_{1}}\right)^{2}\right)+d_{2}(t),
\end{gathered}
$$

where $\widehat{\phi}_{21}\left(\bar{x}_{2}, \widehat{\theta}_{1}, r\right)=\left(\phi_{21}+\left|\partial \alpha_{1} / \partial x_{1}\right| \phi_{11}\right) \tanh \left(z_{2}\left(\phi_{21}+\right.\right.$ $\left.\left.\left|\partial \alpha_{1} / \partial x_{1}\right| \phi_{11}\right) / \epsilon_{21}\right), \epsilon_{21}^{\prime}=0.2785 \epsilon_{21}, \bar{\phi}_{22}\left(x_{1}, \hat{\theta}_{1}, r\right)=\phi_{22}$ 。 $\alpha_{1}^{-1}(2 r)+\left|\partial \alpha_{1} / \partial x_{1}\right| \phi_{12} \circ \alpha_{1}^{-1}(2 r), \widehat{\phi}_{22}\left(x_{1}, \hat{\theta}_{1}, r\right)=\bar{\phi}_{22}\left(x_{1}\right.$, $\left.\hat{\theta}_{1}, r\right) \tanh \left(z_{2} \bar{\phi}_{22}\left(x_{1}, \hat{\theta}_{1}, r\right) / \epsilon_{22}\right)$, and $d_{2}(t)=\sum_{j=1}^{2}\left(\phi_{j 2}\right.$ 。 $\left.\alpha_{1}^{-1}(2 D(t))\right)^{2}$.
Substituting (35) into (33) gives

$$
\begin{aligned}
\dot{V}_{2} \leq & -b k_{1} z_{1}^{2}-\frac{b \sigma_{1} \widetilde{\theta}_{1}^{2}}{2 \gamma_{1}}-\frac{\bar{c}}{\lambda_{0}} r \\
+ & z_{2}\left[g_{2}\left(\bar{x}_{2}\right) x_{3}+g_{1}\left(x_{1}\right) z_{1}+f_{2}\left(\bar{x}_{2}\right)\right. \\
& -\frac{\partial \alpha_{1}}{\partial x_{1}} f_{1}\left(x_{1}\right)-\frac{\partial \alpha_{1}}{\partial x_{1}} g_{1}\left(x_{1}\right) x_{2}-\frac{\partial \alpha_{1}}{\partial \widehat{\theta}_{1}} \\
& -\frac{\partial \alpha_{1}}{\partial r} \dot{r}+\widehat{\phi}_{21}\left(\bar{x}_{2}, \widehat{\theta}_{1}, r\right)+\widehat{\phi}_{22}\left(x_{1}, \widehat{\theta}_{1}, r\right) \\
& \left.+\frac{z_{2}}{4}\left(1+\left(\frac{\partial \alpha_{1}}{\partial x_{1}}\right)^{2}\right)\right]+\epsilon_{21}^{\prime}+\epsilon_{22}^{\prime}+d_{2}(t) \\
& +D_{1}+\frac{d_{0}}{\lambda_{0}}-\frac{b}{\gamma_{2}} \widetilde{\theta}_{2} \dot{\hat{\theta}}_{2} \\
\leq & -b k_{1} z_{1}^{2}-\frac{b \sigma_{1} \widetilde{\theta}_{1}^{2}}{2 \gamma_{1}}-\frac{\bar{c}_{\lambda_{0}}}{\lambda_{0}} \\
+ & z_{2}\left[g_{2}\left(\bar{x}_{2}\right) z_{3}+g_{2}\left(\bar{x}_{2}\right) \alpha_{2}+\widehat{f}_{2}\left(Z_{2}\right)\right]-\frac{z_{2}^{2}}{2}+\epsilon_{21}^{\prime} \\
+ & +\epsilon_{22}^{\prime}+d_{2}(t)+D_{1}+\frac{d_{0}}{\lambda_{0}}-\frac{b}{\gamma_{2}} \widetilde{\theta}_{2} \dot{\hat{\theta}}_{2},
\end{aligned}
$$

where $z_{3}=x_{3}-\alpha_{2}$ and the function $\widehat{f}_{2}\left(Z_{2}\right)$ is specified as

$$
\begin{aligned}
\widehat{f}_{2}\left(Z_{2}\right)= & g_{1}\left(x_{1}\right) z_{1}+f_{2}\left(\bar{x}_{2}\right)-\frac{\partial \alpha_{1}}{\partial x_{1}} f_{1}\left(x_{1}\right)+\widehat{\phi}_{21}\left(x_{1}, \widehat{\theta}_{1}, r\right) \\
& +\widehat{\phi}_{22}\left(\bar{x}_{2}, \widehat{\theta}_{1}, r\right)+\frac{z_{2}}{4}\left[1+\left(\frac{\partial \alpha_{1}}{\partial x_{1}}\right)^{2}\right]+\frac{z_{2}}{2} \\
& -\frac{\partial \alpha_{1}}{\partial x_{1}} g_{1}\left(x_{1}\right) x_{2}-\frac{\partial \alpha_{1}}{\partial \widehat{\theta}_{1}} \dot{\hat{\theta}}_{1}-\frac{\partial \alpha_{1}}{\partial r} \dot{r}
\end{aligned}
$$

with $Z_{2}=\left[\bar{x}_{2}, \hat{\theta}_{1}, r\right]^{T} \in \Omega_{Z_{2}} \in R^{4}$ and $\Omega_{Z_{2}}$ being some known compact set in $R^{4}$.

To compensate for the unknown nonlinear function $\widehat{f}_{2}\left(Z_{2}\right)$, a neural network $\phi_{2}^{T} \xi_{2}\left(Z_{2}\right)$ is utilized to model $\widehat{f}_{2}\left(Z_{2}\right)$ such that, for any given positive constant $\epsilon_{23}$,

$$
\widehat{f}_{2}\left(Z_{2}\right)=\phi_{2}^{T} \xi_{2}\left(Z_{2}\right)+\delta_{2}\left(Z_{2}\right), \quad\left|\delta_{2}\left(Z_{2}\right)\right| \leq \epsilon_{23},
$$

where $\epsilon_{23}$ denotes approximation error.

Then, substituting (38) into (36), one has

$\dot{V}_{2} \leq-b k_{1} z_{1}^{2}-\frac{b \sigma_{1} \widetilde{\theta}_{1}^{2}}{2 \gamma_{1}}-\frac{\bar{c}}{\lambda_{0}} r+g_{2}\left(\bar{x}_{2}\right) z_{2} z_{3}+g_{2}\left(\bar{x}_{2}\right) z_{2} \alpha_{2}$ 


$$
\begin{aligned}
& +z_{2} \phi_{2}^{T} \xi_{2}\left(Z_{2}\right)+z_{2} \delta_{2}\left(Z_{2}\right)-\frac{z_{2}^{2}}{2}+\epsilon_{21}^{\prime}+\epsilon_{22}^{\prime}+d_{2}(t) \\
& +D_{1}+\frac{d_{0}}{\lambda_{0}}-\frac{b}{\gamma_{2}} \widetilde{\theta}_{2} \dot{\hat{\theta}}_{2}
\end{aligned}
$$

By using

$$
\begin{gathered}
z_{2} \phi_{2}^{T} \xi_{2}\left(Z_{2}\right) \leq \frac{b \theta_{2}}{2 \eta_{2}^{2}} \xi_{2}^{T}\left(Z_{2}\right) \xi_{2}\left(Z_{2}\right) z_{2}^{2}+\frac{\eta_{2}^{2}}{2}, \\
z_{2} \delta_{2}\left(Z_{2}\right) \leq \frac{z_{2}^{2}}{2}+\frac{\epsilon_{23}^{2}}{2}
\end{gathered}
$$

it can be easily verified that

$$
\begin{aligned}
\dot{V}_{2} \leq & -b k_{1} z_{1}^{2}-\frac{b \sigma_{1} \widetilde{\theta}_{1}^{2}}{2 \gamma_{1}}-\frac{\bar{c}}{\lambda_{0}} r+g_{2}\left(\bar{x}_{2}\right) z_{2} \alpha_{2} \\
& +\frac{b \theta_{2}}{2 \eta_{2}^{2}} \xi_{2}^{T}\left(Z_{2}\right) \xi_{2}\left(Z_{2}\right) z_{2}^{2}+g_{2}\left(\bar{x}_{2}\right) z_{2} z_{3} \\
& +\frac{\eta_{2}^{2}}{2}+\epsilon_{21}^{\prime}+\epsilon_{22}^{\prime}+\frac{\epsilon_{23}^{2}}{2}+d_{2}(t)+D_{1}+\frac{d_{0}}{\lambda_{0}}-\frac{b}{\gamma_{2}} \tilde{\theta}_{2} \dot{\hat{\theta}}_{2}
\end{aligned}
$$

with $\theta_{2}=b^{-1}\left\|\phi_{2}\right\|^{2}$ being an unknown constant.

Furthermore, the virtual control $\alpha_{2}$ is constructed as

$$
\alpha_{2}=-k_{2} z_{2}-\frac{\widehat{\theta}_{2}}{2 \eta_{2}^{2}} z_{2} \xi_{2}^{T}\left(Z_{2}\right) \xi_{2}\left(Z_{2}\right)
$$

where $k_{2}>0$ and $\eta_{2}$ are the design constants.

Then, the following result can be easily obtained:

$$
g_{2}\left(\bar{x}_{2}\right) z_{2} \alpha_{2} \leq-k_{2} b z_{2}^{2}-\frac{\widehat{\theta}_{2}}{2 \eta_{2}^{2}} b z_{2}^{2} \xi_{2}^{T}\left(Z_{2}\right) \xi_{2}\left(Z_{2}\right)
$$

By applying (43), (41) can be rewritten as

$$
\begin{aligned}
\dot{V}_{2} \leq & -\sum_{i=1}^{2} k_{i} b z_{i}^{2}-\frac{b \sigma_{1} \widetilde{\theta}_{1}^{2}}{2 \gamma_{1}}-\frac{\bar{c}}{\lambda_{0}} r \\
& +\frac{b \widetilde{\theta}_{2}}{\gamma_{2}}\left(\frac{\gamma_{2}}{2 \eta_{2}^{2}} \xi_{2}^{T}\left(Z_{2}\right) \xi_{2}\left(Z_{2}\right) z_{2}^{2}-\dot{\hat{\theta}}_{2}\right)+g_{2}\left(\bar{x}_{2}\right) z_{2} z_{3} \\
& +\frac{\eta_{2}^{2}}{2}+\epsilon_{21}^{\prime}+\epsilon_{22}^{\prime}+\frac{\epsilon_{23}^{2}}{2}+d_{2}(t)+\frac{d_{0}}{\lambda_{0}}+D_{1} .
\end{aligned}
$$

Define the adaptive law as

$$
\dot{\hat{\theta}}_{2}=\frac{\gamma_{2}}{2 \eta_{2}^{2}} \xi_{2}^{T}\left(Z_{2}\right) \xi_{2}\left(Z_{2}\right) z_{2}^{2}-\sigma_{2} \widehat{\theta}_{2}
$$

where $\eta_{2}, \gamma_{2}$, and $\sigma_{2}$ are design parameters.
Combining (44) with (45) produces

$$
\begin{aligned}
\dot{V}_{2} \leq & -\sum_{i=1}^{2} k_{i} b z_{i}^{2}-\frac{b \sigma_{1} \widetilde{\theta}_{1}^{2}}{2 \gamma_{1}}-\frac{\bar{c}}{\lambda_{0}} r+\frac{b \sigma_{2} \widetilde{\theta}_{2} \widehat{\theta}_{2}}{\gamma_{2}}+g_{2}\left(\bar{x}_{2}\right) z_{2} z_{3} \\
& +\frac{\eta_{2}^{2}}{2}+\epsilon_{21}^{\prime}+\epsilon_{22}^{\prime}+\frac{\epsilon_{23}^{2}}{2}+d_{2}(t)+D_{1}+\frac{d_{0}}{\lambda_{0}} \\
\leq & -\sum_{i=1}^{2}\left(k_{i} b z_{i}^{2}+\frac{b \sigma_{i} \widetilde{\theta}_{i}^{2}}{2 \gamma_{i}}\right)-\frac{\bar{c}}{\lambda_{0}} r+\frac{d_{0}}{\lambda_{0}} \\
& +\sum_{i=1}^{2} D_{i}+g_{2}\left(\bar{x}_{2}\right) z_{2} z_{3},
\end{aligned}
$$

where $D_{i}=\eta_{i}^{2} / 2+b \sigma_{i} \theta_{i}^{2} / 2 \gamma_{i}+\epsilon_{i 3}^{2} / 2+\epsilon_{i 1}^{\prime}+\epsilon_{i 2}^{\prime}+d_{i}(t), i=1,2$, and the result $b \sigma_{2} \widetilde{\theta}_{2} \widehat{\theta}_{2} / \gamma_{2} \leq-b \sigma_{2} \widetilde{\theta}_{2}^{2} / 2 \gamma_{2}+b \sigma_{2} \theta_{2}^{2} / 2 \gamma_{2}$ has been used in the above equation.

Step $i(3 \leq i \leq n-1)$. According to $z_{i}=x_{i}-\alpha_{i-1}$, the dynamics of $z_{i}$ is

$$
\begin{aligned}
\dot{z}_{i}= & g_{i}\left(\bar{x}_{i}\right) x_{i+1}+f_{i}\left(\bar{x}_{i}\right)+\Delta_{i} \\
& -\sum_{j=1}^{i-1} \frac{\partial \alpha_{i-1}}{\partial x_{j}}\left(g_{j}\left(\bar{x}_{j}\right) x_{j+1}+f_{j}\left(\bar{x}_{j}\right)+\Delta_{j}\right) \\
& -\sum_{j=1}^{i-1} \frac{\partial \alpha_{i-1}}{\partial \hat{\theta}_{j}} \dot{\hat{\theta}}_{j}-\frac{\partial \alpha_{i-1}}{\partial r} \dot{r} \\
= & g_{i}\left(\bar{x}_{i}\right) x_{i+1}+f_{i}\left(\bar{x}_{i}\right)-\sum_{j=1}^{i-1} \frac{\partial \alpha_{i-1}}{\partial x_{j}}\left(g_{j}\left(\bar{x}_{j}\right) x_{j+1}+f_{j}\left(\bar{x}_{j}\right)\right) \\
& +\bar{\Delta}_{i}-\sum_{j=1}^{i-1} \frac{\partial \alpha_{i-1}}{\partial \widehat{\theta}_{j}} \dot{\hat{\theta}}_{j}-\frac{\partial \alpha_{i-1}}{\partial r} \dot{r}
\end{aligned}
$$

where $\bar{\Delta}_{i}=\Delta_{i}-\sum_{j=1}^{i-1}\left(\partial \alpha_{i-1} / \partial x_{j}\right) \Delta_{j}$

Consider the Lyapunov function $V_{i}$ as

$$
V_{i}=V_{i-1}+\frac{1}{2} z_{i}^{2}+\frac{b}{2 \gamma_{i}} \widetilde{\theta}_{i}^{2}
$$

By using the derivations similar to those used in the former steps, we can obtain

$$
\begin{aligned}
\dot{V}_{i} \leq & -\sum_{j=1}^{i-1}\left(k_{j} b z_{j}^{2}+\frac{b \sigma_{j} \widetilde{\theta}_{j}^{2}}{2 \gamma_{j}}\right)-\frac{\bar{c}}{\lambda_{0}} r \\
& +z_{i}\left(g_{i}\left(\bar{x}_{i}\right) x_{i+1}+f_{i}\left(\bar{x}_{i}\right)-\sum_{j=1}^{i-1} \frac{\partial \alpha_{i-1}}{\partial x_{j}} f_{j}\left(\bar{x}_{j}\right)\right. \\
& -\sum_{j=1}^{i-1} \frac{\partial \alpha_{i-1}}{\partial x_{j}} g_{j}\left(\bar{x}_{j}\right) x_{j+1}-\sum_{j=1}^{i-1} \frac{\partial \alpha_{i-1}}{\partial \widehat{\theta}_{j}} \dot{\hat{\theta}}_{j}
\end{aligned}
$$




$$
\begin{array}{r}
\left.-\frac{\partial \alpha_{i-1}}{\partial r} \dot{r}+g_{i-1}\left(\bar{x}_{i-1}\right) z_{i-1}\right) \\
+\left|z_{i} \bar{\Delta}_{i}\right|+\frac{d_{0}}{\lambda_{0}}+\sum_{j=1}^{i-1} D_{j}-\frac{b}{\gamma_{i}} \widetilde{\theta}_{i} \dot{\hat{\theta}}_{i} .
\end{array}
$$

Similar to (34), we have

$$
\begin{aligned}
\left|z_{i} \bar{\Delta}_{i}\right| \leq & \left|z_{i}\right|\left(\left|\Delta_{i}\right|+\sum_{j=1}^{i-1}\left|\frac{\partial \alpha_{i-1}}{\partial x_{j}}\right|\left|\Delta_{j}\right|\right) \\
\leq & \left|z_{i}\right|\left(\phi_{i 1}+\sum_{j=1}^{i-1}\left|\frac{\partial \alpha_{i-1}}{\partial x_{j}}\right| \phi_{j 1}\right) \\
& +\left|z_{i}\right|\left(\phi_{i 2}(|z|)+\sum_{j=1}^{i-1}\left|\frac{\partial \alpha_{i-1}}{\partial x_{j}}\right| \phi_{j 2}(|z|)\right) .
\end{aligned}
$$

Furthermore, the following inequalities can be easily verified by repeating the same arguments as (35):

$$
\begin{gathered}
\left|z_{i}\right|\left(\phi_{i 1}+\sum_{j=1}^{i-1}\left|\frac{\partial \alpha_{i-1}}{\partial x_{j}}\right| \phi_{j 1}\right) \leq z_{i} \widehat{\phi}_{i 1}\left(\bar{x}_{i}, \overline{\hat{\theta}}_{i-1}, r\right)+\epsilon_{i 1}^{\prime}, \\
\left|z_{i}\right|\left(\phi_{i 2}(|z|)+\sum_{j=1}^{i-1}\left|\frac{\partial \alpha_{i-1}}{\partial x_{j}}\right| \phi_{j 2}(|z|)\right) \\
\leq z_{i} \widehat{\phi}_{i 2}\left(\bar{x}_{i}, \overline{\widehat{\theta}}_{i-1}, r\right)+\frac{z_{i}^{2}}{4}\left[1+\sum_{j=1}^{i-1}\left(\frac{\partial \alpha_{i-1}}{\partial x_{j}}\right)^{2}\right] \\
+\epsilon_{i 2}^{\prime}+d_{i}(t)
\end{gathered}
$$

where $\widehat{\phi}_{i 1}\left(\bar{x}_{i}, \overline{\widehat{\theta}}_{i-1}, r\right)=\left(\phi_{i 1}+\sum_{j=1}^{i-1}\left|\partial \alpha_{i-1} / \partial x_{j}\right| \phi_{j 1}\right) \tanh \left(z_{i}\left(\phi_{i 1}\right.\right.$ $\left.\left.+\sum_{j=1}^{i-1}\left|\partial \alpha_{i-1} / \partial x_{j}\right| \phi_{j 1}\right) / \epsilon_{i 1}\right), \epsilon_{i 1}^{\prime}=0.2785 \epsilon_{i 1}, \widehat{\phi}_{i 2}\left(\bar{x}_{i}, \overline{\widehat{\theta}}_{i-1}, r\right)=$ $\bar{\phi}_{i 2}\left(\bar{x}_{i}, \overline{\hat{\theta}}_{i-1}, r\right) \tanh \left(z_{i} \bar{\phi}_{i 2}\left(\bar{x}_{i}, \overline{\hat{\theta}}_{i-1}, r\right) / \epsilon_{i 2}\right), \bar{\phi}_{i 2}\left(\bar{x}_{i}, \overline{\hat{\theta}}_{i-1}, r\right)=\phi_{i 2}$ 。 $\alpha_{1}^{-1}(2 r)+\sum_{j=1}^{i-1}\left|\partial_{i-1} / \partial x_{j}\right| \phi_{j 2} \circ \alpha_{1}^{-1}(2 r), \epsilon_{i 2}^{\prime}=0.2785 \epsilon_{i 2}$, and $d_{i}(t)=\sum_{j=1}^{i}\left(\phi_{j 2} \circ \alpha_{1}^{-1}(2 D(t))\right)^{2}$, noting that $d_{i}(t) \geq 0$ for all $t \geq 0$.

Substituting (51) and (52) into (49) results in

$$
\begin{aligned}
\dot{V}_{i} \leq & -\sum_{j=1}^{i-1}\left(k_{j} b z_{j}^{2}+\frac{b \sigma_{j} \widetilde{\theta}_{j}^{2}}{2 \gamma_{j}}\right)-\frac{\bar{c}}{\lambda_{0}} r \\
& +z_{i}\left(g_{i}\left(\bar{x}_{i}\right) z_{i+1}+g_{i}\left(\bar{x}_{i}\right) \alpha_{i}+\widehat{f}_{i}\left(Z_{i}\right)\right) \\
& -\frac{z_{i}^{2}}{2}+\epsilon_{i 1}^{\prime}+\epsilon_{i 2}^{\prime}+d_{i}(t)+\frac{d_{0}}{\lambda_{0}}+\sum_{j=1}^{i-1} D_{j}-\frac{b}{\gamma_{i}} \widetilde{\theta}_{i} \dot{\hat{\theta}}_{i},
\end{aligned}
$$

where the function $\widehat{f}_{i}\left(Z_{i}\right)$ is defined by

$$
\begin{aligned}
\widehat{f}_{i}\left(Z_{i}\right)= & f_{i}\left(\bar{x}_{i}\right)-\sum_{j=1}^{i-1} \frac{\partial \alpha_{i-1}}{\partial x_{j}} f_{j}\left(\bar{x}_{j}\right)-\sum_{j=1}^{i-1} \frac{\partial \alpha_{i-1}}{\partial x_{j}} g_{j}\left(\bar{x}_{j}\right) x_{j+1} \\
& -\sum_{j=1}^{i-1} \frac{\partial \alpha_{i-1}}{\partial \widehat{\hat{\theta}}_{j}}-\frac{\partial \alpha_{i-1}}{\partial r} \dot{r}+g_{i-1}\left(\bar{x}_{i-1}\right) z_{i-1} \\
& +\widehat{\phi}_{i 1}\left(\bar{x}_{i}, \overline{\hat{\theta}}_{i-1}, r\right)+\widehat{\phi}_{i 2}\left(\bar{x}_{i}, \overline{\hat{\theta}}_{i-1}, r\right) \\
& +\frac{z_{i}}{4}\left[1+\sum_{j=1}^{i-1}\left(\frac{\partial \alpha_{i-1}}{\partial x_{j}}\right)^{2}\right]+\frac{z_{i}}{2}
\end{aligned}
$$

where $Z_{i}=\left[\bar{x}_{i}, \overline{\hat{\theta}}_{i-1}, r\right]^{T} \in \Omega_{Z_{i}} \in R^{2 i}, \bar{x}_{i}=\left[x_{1}, \ldots, x_{i}\right]^{T}$, and $\Omega_{Z_{i}}$ is some known compact set in $R^{2 i}$.

Currently, a neural network $\phi_{i}^{T} \xi_{i}\left(Z_{i}\right)$ is utilized to model $\widehat{f}_{i}\left(Z_{i}\right)$ such that, for a given $\epsilon_{i 3}>0, \widehat{f}_{i}\left(Z_{i}\right)$ can be expressed as

$$
\widehat{f}_{i}\left(Z_{i}\right)=\phi_{i}^{T} \xi_{i}\left(Z_{i}\right)+\delta_{i}\left(Z_{i}\right), \quad\left|\delta_{i}\left(Z_{i}\right)\right| \leq \epsilon_{i 3} .
$$

Further, similar to (40), we can obtain

$$
\begin{gathered}
z_{i} \phi_{i}^{T} \xi_{i}\left(Z_{i}\right) \leq \frac{b \theta_{i}}{2 \eta_{i}^{2}} \xi_{i}^{T}\left(Z_{i}\right) \xi_{i}\left(Z_{i}\right) z_{i}^{2}+\frac{\eta_{i}^{2}}{2}, \\
z_{i} \delta_{i}\left(Z_{i}\right) \leq \frac{z_{i}^{2}}{2}+\frac{\epsilon_{i 3}^{2}}{2},
\end{gathered}
$$

where $\theta_{i}=b^{-1}\left\|\phi_{i}\right\|^{2}$ is an unknown constant.

Now, construct the virtual control signal $\alpha_{i}$ as

$$
\alpha_{i}=-k_{i} z_{i}-\frac{\widehat{\theta}_{i}}{2 \eta_{i}^{2}} z_{i} \xi_{i}^{T}\left(Z_{i}\right) \xi_{i}\left(Z_{i}\right)
$$

with $k_{i}>0$ and $\eta_{i}$ being design constants.

Then, by substituting (55)-(57) into (53), choosing the adaptive law

$$
\dot{\hat{\theta}}_{i}=\frac{\gamma_{i}}{2 \eta_{i}^{2}} \xi_{i}^{T}\left(Z_{i}\right) \xi_{i}\left(Z_{i}\right) z_{i}^{2}-\sigma_{i} \widehat{\theta}_{i}
$$

with $\eta_{i}, \gamma_{i}$, and $\sigma_{i}$ being the design parameters, and then following the same line as the procedures from (43) to (46), we have

$$
\begin{aligned}
\dot{V}_{i} \leq & -\sum_{j=1}^{i}\left(k_{j} b z_{j}^{2}+\frac{b \sigma_{j} \widetilde{\theta}_{j}^{2}}{2 \gamma_{j}}\right)-\frac{\bar{c}}{\lambda_{0}} r+\frac{d_{0}}{\lambda_{0}}+\sum_{j=1}^{i} D_{j} \\
& +g_{i}\left(\bar{x}_{i}\right) z_{i} z_{i+1},
\end{aligned}
$$
where $D_{j}=\eta_{j}^{2} / 2+b \sigma_{j} \theta_{j}^{2} / 2 \gamma_{j}+\epsilon_{j 3}^{2} / 2+\epsilon_{j 1}^{\prime}+\epsilon_{j 2}^{\prime}+d_{j}(t), j=$
$1,2, \ldots, i$. 
Step $n$. In this step, the actual controller $u$ is designed. According to $z_{n}=x_{n}-\alpha_{n-1}$, then we have

$$
\begin{aligned}
\dot{z}_{n}= & g_{n}\left(\bar{x}_{n}\right) u+f_{n}\left(\bar{x}_{n}\right)-\sum_{j=1}^{n-1} \frac{\partial \alpha_{n-1}}{\partial x_{j}} f_{j}\left(\bar{x}_{j}\right) \\
& -\sum_{j=1}^{n-1} \frac{\partial \alpha_{n-1}}{\partial x_{j}} g_{j}\left(\bar{x}_{j}\right) x_{j+1}+\bar{\Delta}_{n}-\sum_{j=1}^{n-1} \frac{\partial \alpha_{n-1}}{\partial \hat{\theta}_{j}} \dot{\hat{\theta}}_{j}-\frac{\partial \alpha_{n-1}}{\partial r} \dot{r}
\end{aligned}
$$

where $\bar{\Delta}_{n}=\Delta_{n}-\sum_{i=1}^{n-1}\left(\partial \alpha_{n-1} / \partial x_{i}\right) \Delta_{i}$. Similarly, choose the following Lyapunov function as

$$
V_{n}=V_{n-1}+\frac{1}{2} z_{n}^{2}+\frac{b}{2 \gamma_{n}} \widetilde{\theta}_{n}^{2}
$$

From (53) and (54), we have

$$
\begin{aligned}
\dot{V}_{n} \leq & -\sum_{j=1}^{n-1}\left(k_{j} b z_{j}^{2}+\frac{b \sigma_{j} \widetilde{\theta}_{j}^{2}}{2 \gamma_{j}}\right)-\frac{\bar{c}}{\lambda_{0}} r+\frac{d_{0}}{\lambda_{0}} \\
& +\sum_{j=1}^{n-1} D_{j}+g_{n-1}\left(\bar{x}_{n-1}\right) z_{n-1} z_{n} \\
& +z_{n}\left(g_{n}\left(\bar{x}_{n}\right) u+f_{n}\left(\bar{x}_{n}\right)-\sum_{i=1}^{n-1} \frac{\partial \alpha_{n-1}}{\partial x_{i}} f_{i}\left(\bar{x}_{i}\right)\right. \\
& -\sum_{i=1}^{n-1} \frac{\partial \alpha_{n-1}}{\partial x_{i}} g_{i}\left(\bar{x}_{i}\right) x_{i+1}-\sum_{i=1}^{n-1} \frac{\partial \alpha_{n-1}}{\partial \widehat{\theta}_{i}} \\
& \left.-\frac{\partial \alpha_{n-1}}{\partial r} \dot{r}\right)-\frac{b}{\gamma_{n}} \tilde{\theta}_{n} \dot{\hat{\theta}}_{n}+\left|z_{n} \bar{\Delta}_{n}\right| .
\end{aligned}
$$

Using the same estimation methods as (42)-(44), we have

$$
\begin{aligned}
\left|z_{n} \bar{\Delta}_{n}\right| \leq & z_{n} \widehat{\phi}_{n 1}\left(\bar{x}_{n}, \overline{\hat{\theta}}_{n-1}, r\right)+\epsilon_{n 1}^{\prime}+z_{n} \widehat{\phi}_{n 2}\left(\bar{x}_{n}, \overline{\hat{\theta}}_{n-1}, r\right) \\
+ & \frac{z_{n}^{2}}{4}\left[1+\sum_{j=1}^{n-1}\left(\frac{\partial \alpha_{n-1}}{\partial x_{j}}\right)^{2}\right]+\epsilon_{n 2}^{\prime}+d_{n}(t),
\end{aligned}
$$

where $\widehat{\phi}_{n 1}\left(\bar{x}_{n}, \overline{\widehat{\theta}}_{n-1}, r\right), \epsilon_{n 1}^{\prime}, \widehat{\phi}_{n 2}\left(\bar{x}_{n}, \overline{\hat{\theta}}_{n-1}, r\right), \epsilon_{n 2}^{\prime}$, and $d_{n}(t)$ are defined in (51) or (52) with $i=n$. By substituting (63) into (62), one has

$$
\begin{aligned}
\dot{V}_{n} \leq & -\sum_{j=1}^{n-1}\left(k_{j} b z_{j}^{2}+\frac{b \sigma_{j} \widetilde{\theta}_{j}^{2}}{2 \gamma_{j}}\right)-\frac{\bar{c}}{\lambda_{0}} r+\frac{d_{0}}{\lambda_{0}}+\sum_{j=1}^{n-1} D_{j}+\epsilon_{n 1}^{\prime} \\
& +\epsilon_{n 2}^{\prime}+d_{n}(t)+z_{n}\left(g_{n}\left(\bar{x}_{n}\right) u+\widehat{f}_{n}\left(Z_{n}\right)\right) \\
& -\frac{z_{n}^{2}}{2}-\frac{b}{\gamma_{n}} \widetilde{\theta}_{n} \dot{\hat{\theta}}_{n}
\end{aligned}
$$

where the function $\widehat{f}_{n}\left(Z_{n}\right)$ is defined by

$$
\begin{aligned}
\widehat{f}_{n}\left(Z_{n}\right)= & g_{n-1}\left(\bar{x}_{n-1}\right) z_{n-1}+f_{n}\left(\bar{x}_{n}\right)-\sum_{i=1}^{n-1} \frac{\partial \alpha_{n-1}}{\partial x_{i}} f_{i}\left(\bar{x}_{i}\right) \\
& -\sum_{i=1}^{n-1} \frac{\partial \alpha_{n-1}}{\partial x_{i}} g_{i}\left(\bar{x}_{i}\right) x_{i+1}-\sum_{i=1}^{n-1} \frac{\partial \alpha_{n-1}}{\partial \widehat{\hat{\theta}}_{i}}-\frac{\partial \alpha_{n-1}}{\partial r} \dot{r} \\
& +\widehat{\phi}_{n 1}\left(\bar{x}_{n}, \overline{\widehat{\theta}}_{n-1}, r\right)+\widehat{\phi}_{n 2}\left(\bar{x}_{n}, \overline{\hat{\theta}}_{n-1}, r\right) \\
& +\frac{z_{n}}{4}\left[1+\sum_{j=1}^{n-1}\left(\frac{\partial \alpha_{n-1}}{\partial x_{j}}\right)^{2}\right]+\frac{z_{n}}{2}
\end{aligned}
$$

with $Z_{n}=\left[\bar{x}_{n}, \overline{\hat{\theta}}_{n-1}, r\right]^{T} \in \Omega_{Z_{n}} \in R^{2 n}$ and $\Omega_{Z_{n}}$ being some known compact set in $R^{2 n}$. Similarly, neural network $\phi_{n}^{T} \xi_{n}\left(Z_{n}\right)$ is employed to model $\widehat{f}_{n}\left(Z_{n}\right)$ such that $\widehat{f}_{n}\left(Z_{n}\right)=$ $\phi_{n}^{T} \xi_{n}\left(Z_{n}\right)+\delta_{n}\left(Z_{n}\right),\left|\delta_{n}\left(Z_{n}\right)\right| \leq \epsilon_{n 3}$. Then, following the same line as used in (40), we have

$$
\begin{aligned}
z_{n} \phi_{n}^{T} \xi_{n}\left(Z_{n}\right) & \leq \frac{b \theta_{n}}{2 \eta_{n}^{2}} \xi_{n}^{T}\left(Z_{n}\right) \xi_{n}\left(Z_{n}\right) z_{n}^{2}+\frac{\eta_{n}^{2}}{2}, \\
z_{n} \delta_{n}\left(Z_{n}\right) & \leq \frac{z_{n}^{2}}{2}+\frac{\epsilon_{n 3}^{2}}{2},
\end{aligned}
$$

where $\theta_{n}=b^{-1}\left\|\phi_{n}\right\|^{2}$ denotes an unknown constant and $\eta_{n}$ is a design constant.

Subsequently, by combining (64) together with (66), the inequality below holds:

$$
\begin{aligned}
\dot{V}_{n} \leq & -\sum_{j=1}^{n-1}\left(k_{j} b z_{j}^{2}+\frac{b \sigma_{j} \widetilde{\theta}_{j}^{2}}{2 \gamma_{j}}\right)-\frac{\bar{c}}{\lambda_{0}} r+\frac{d_{0}}{\lambda_{0}}+\sum_{j=1}^{n-1} D_{j} \\
& +\epsilon_{n 1}^{\prime}+\epsilon_{n 2}^{\prime}+d_{n}(t)+z_{n} g_{n}\left(\bar{x}_{n}\right) u \\
& +\frac{b \theta_{n}}{2 \eta_{n}^{2}} \xi_{n}^{T}\left(Z_{n}\right) \xi_{n}\left(Z_{n}\right) z_{n}^{2}+\frac{\eta_{n}^{2}}{2}+\frac{\epsilon_{n 3}^{2}}{2}-\frac{b}{\gamma_{n}} \tilde{\theta}_{n} \dot{\hat{\theta}}_{n} .
\end{aligned}
$$

At the present stage, construct the real controller $u$ and adaptive law $\dot{\hat{\theta}}_{n}$ in the following forms:

$$
\begin{gathered}
u=-k_{n} z_{n}-\frac{\widehat{\theta}_{n}}{2 \eta_{n}^{2}} z_{n} \xi_{n}^{T}\left(Z_{n}\right) \xi_{n}\left(Z_{n}\right), \\
\dot{\hat{\theta}}_{n}=\frac{\gamma_{n}}{2 \eta_{n}^{2}} \xi_{n}^{T}\left(Z_{n}\right) \xi_{i}\left(Z_{n}\right) z_{n}^{2}-\sigma_{n} \widehat{\theta}_{n},
\end{gathered}
$$

where $k_{n}, \eta_{n}, \gamma_{n}$, and $\sigma_{n}$ are design constants.

Then, repeating the similar procedures as (43)-(46), we can obtain

$$
\dot{V}_{n} \leq-\sum_{j=1}^{n}\left(k_{j} b z_{j}^{2}+\frac{b \sigma_{j} \widetilde{\theta}_{j}^{2}}{2 \gamma_{j}}\right)-\frac{\bar{c}}{\lambda_{0}} r+\frac{d_{0}}{\lambda_{0}}+\sum_{j=1}^{n} D_{j},
$$

where $D_{j}=\eta_{j}^{2} / 2+b \sigma_{j} \theta_{j}^{2} / 2 \gamma_{j}+\epsilon_{j 3}^{2} / 2+\epsilon_{j 1}^{\prime}+\epsilon_{j 2}^{\prime}+d_{j}(t), j=$ $1,2, \ldots, n$. 
Now, the main result of this research is summarized as follows.

Theorem 8. Consider the system (1) consisting of Assumptions $2-5$, the control input (68), and the adaptive laws (58) and (69). Assume that the packaged unknown functions $\widehat{f}_{i}\left(Z_{i}\right)(i=$ $1,2, \ldots, n)$ could be modeled by neural networks $\phi_{i}^{T} \xi_{i}\left(Z_{i}\right)$ with the bounded approximation errors. Then, for bounded initial values with $\widehat{\theta}_{i}(0) \geq 0$, all the signals in the closed-loop system are semiglobally boundedness in mean square.

Proof. To give the stability analysis for the closed-loop system, consider the Lyapunov function in the form $V=V_{n}$, and define

$$
a_{0}=\min \left\{2 k_{i} b, \bar{c}, \sigma_{i}, i=1,2, \ldots, n\right\}, \quad b_{0}=\frac{d_{0}}{\lambda_{0}}+\sum_{j=1}^{n} D_{j} .
$$

Furthermore, we can rewrite (70) as

$$
\dot{V} \leq-a_{0} V+b_{0} .
$$

Next, from (72), the following inequality can be easily verified:

$$
V(t) \leq\left(V(0)-\frac{b_{0}}{a_{0}}\right) e^{-a_{0} t}+\frac{b_{0}}{a_{0}}, \quad \forall t>0,
$$

which means that

$$
V(t) \leq V(0)+\frac{b_{0}}{a_{0}}, \quad \forall t>0 .
$$

Therefore, based on the definition of $V$ in $(61), z_{j}, \widetilde{\theta}_{j}$ $(j=1,2, \ldots, n)$, and $r$ are bounded. Because the signal $r$ is bounded, the trajectory $z(t)$ is bounded. Since $\theta_{i}$ are constants, $\widehat{\theta}_{i}$ are bounded. Consequently, $\alpha_{i}$ are also bounded because $z_{i}$ and $\widehat{\theta}_{i}$ are bounded variables. Hence, we conclude that the signals $x_{i}$ are bounded.

\section{Simulation Example}

A simulation example is presented to show the effectiveness of the proposed control scheme. Consider the second-order nonlinear system as

$$
\begin{aligned}
& \dot{z}=-z+x_{1}^{2}+0.5, \\
& \dot{x}_{1}=x_{2}+x_{1}^{2} e^{-0.5 x_{1}}+z x_{1} \sin \left(x_{1}\right), \\
& \dot{x}_{2}=u+x_{1} x_{2}^{2}+x_{1} x_{2} z,
\end{aligned}
$$

where $f_{1}\left(x_{1}\right)=x_{1}^{2} e^{-0.5 x_{1}}, f_{2}\left(x_{1}, x_{2}\right)=x_{1} x_{2}^{2}, \Delta_{1}=$ $z x_{1} \sin \left(x_{1}\right)$, and $\Delta_{2}=x_{1} x_{2} z$. It can be easily verified that Assumption 2 is satisfied. In order to check Assumption 4 holds for $z$-subsystem in (75), consider $V_{z}(z)=z^{2}$, and then

$$
\begin{aligned}
\dot{V}_{z}(z) & =2 z\left(-z+x_{1}^{2}+0.5\right) \\
& \leq-2 z^{2}+\frac{1}{4 \varepsilon}(2 z)^{2}+\varepsilon x_{1}^{4}+\frac{\varepsilon}{4}+\frac{z^{2}}{\varepsilon} .
\end{aligned}
$$

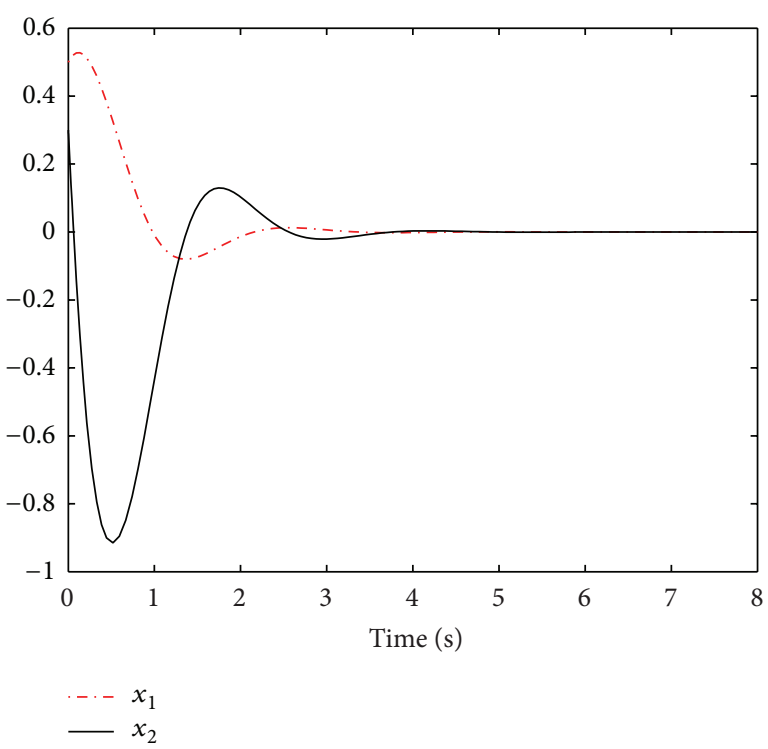

Figure 1: State variables $x_{1}$ and $x_{2}$.

By choosing $\varepsilon=2.5$, we have

$$
\dot{V}_{z}(z) \leq-1.2 z^{2}+2.5 x_{1}^{4}+0.625 .
$$

By Defining $\alpha_{1}(|z|)=0.5 z^{2}, \alpha_{2}(|z|)=2 z^{2}, c_{0}=1.2, d_{0}=$ 0.625 , and $\mu\left(\left|x_{1}\right|\right)=2.5 x_{1}^{4}$, Assumption 4 is satisfied. Take $\bar{c}=1 \in\left(0, c_{0}\right)$ and define the dynamic signal as follows:

$$
\dot{r}=-r+2.5 x_{1}^{4}+0.625 \text {. }
$$

By using Theorem 8 , the virtual control signal, the real control input, and the adaptive laws are constructed as follows:

$$
\begin{gathered}
\alpha_{1}=-k_{1} z_{1}-\frac{\widehat{\theta}_{1}}{2 \eta_{1}^{2}} \xi_{1}^{T}\left(Z_{1}\right) \xi_{2}\left(Z_{1}\right) z_{1}, \\
u=-k_{2} z_{2}-\frac{\widehat{\theta}_{2}}{2 \eta_{2}^{2}} \xi_{2}^{T}\left(Z_{2}\right) \xi_{2}\left(Z_{2}\right) z_{2}, \\
\dot{\hat{\theta}}_{i}=\frac{\gamma_{i}}{2 \eta_{i}^{2}} \xi_{i}^{T}\left(Z_{i}\right) \xi_{i}\left(Z_{i}\right) z_{i}^{2}-\sigma_{i} \widehat{\theta}_{i}, \quad i=1,2 .
\end{gathered}
$$

In the simulation, the design constants are chosen as $k_{1}=$ $k_{2}=3, \eta_{1}=\eta_{2}=1, \gamma_{1}=\gamma_{2}=2$, and $\sigma_{1}=\sigma_{2}=$ 1. The simulation is carried out with the initial conditions $\left[x_{1}(0), x_{2}(0), \widehat{\theta}_{1}(0), \widehat{\theta}_{2}(0)\right]^{T}=[0.5,0.3,0,0]$.

The simulation results are shown in Figures 1-3. Figure 1 shows the trajectories of states $x_{1}$ and $x_{2}$. Figure 2 displays the trajectory of control input $u$. Figure 3 shows the trajectories of adaptive parameters $\widehat{\theta}_{1}$ and $\hat{\theta}_{2}$. From Figures $1-3$, we can see that the proposed control approach can guarantee the boundedness of the variables $x_{1}, x_{2}, u, \widehat{\theta}_{1}$, and $\widehat{\theta}_{2}$.

\section{Conclusion}

In this research, a backstepping-based adaptive neural control scheme has been developed for strict-feedback nonlinear systems with unmodeled dynamics and dynamic disturbances. 


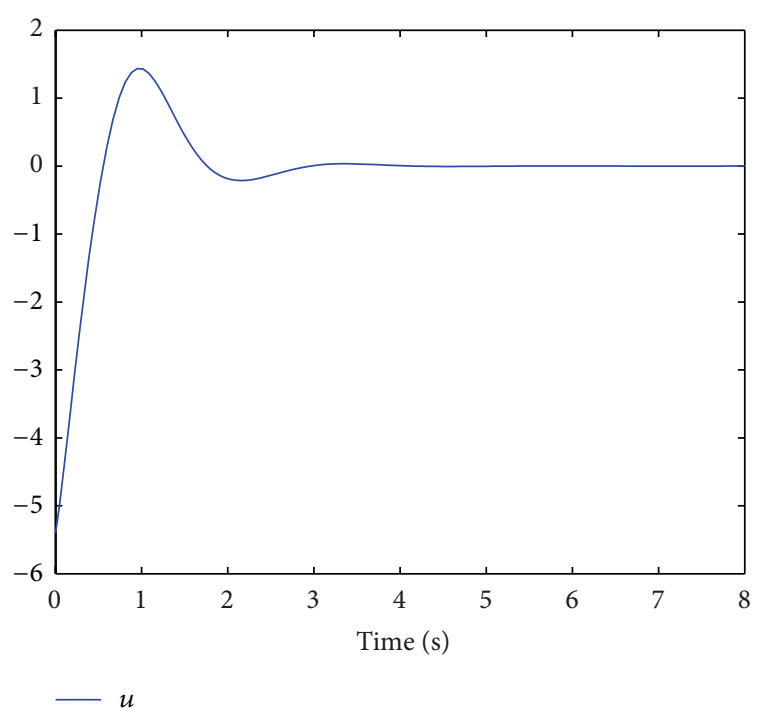

Figure 2: The actual control input $u$.

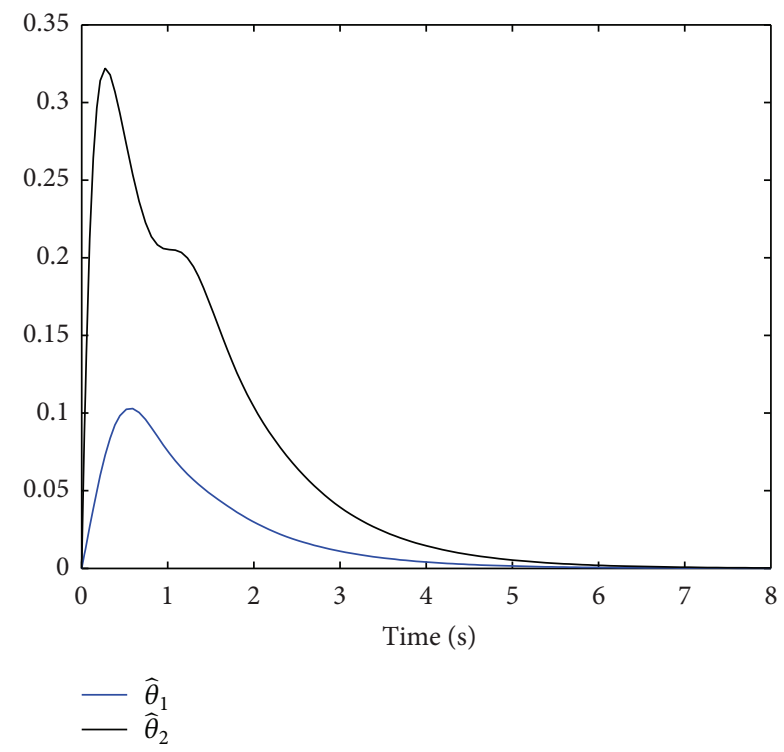

FIgURE 3: The adaptive parameters $\widehat{\theta}_{1}$ and $\widehat{\theta}_{2}$.

The proposed adaptive neural controller guarantees that all the signals of the resulting closed-loop system remain semiglobally uniformly ultimately bounded in the sense of mean square. Simulation results have been provided to illustrate the effectiveness of the proposed control scheme. It should be pointed out that the work in this paper does not consider the problem of input nonlinearity and time-delay. Then, they may occur in practical engineering. So, how to control a nonlinear system with input nonlinearity and time-delay is our future research direction.

\section{Conflict of Interests}

The authors declare that there is no conflict of interests regarding the publication of this paper.

\section{Acknowledgments}

This work is partially supported by the Natural Science Foundation of China (61304002, 61304102, and 61304003), the Program for New Century Excellent Tallents in University (NECT-13-0696), the Program for Liaoning Innovative Research Team in University under Grant (LT2013023), the Program for Liaoning Excellent Talents in University under Grant (LR2013053), and the Education Department of Liaoning Province under the general project research under Grant (no. L2013424).

\section{References}

[1] I. Kanellakopoulos, P. V. Kokotovic, and A.S. Morse, "Systematic design of adaptive controllers for feedback linearizable systems," IEEE Transactions on Automatic Control, vol. 36, no. 11, pp. 12411253, 1991.

[2] M. Krstić, I. Kanellakopoulos, and P. V. Kokotović, "Adaptive nonlinear control without overparametrization," Systems and Control Letters, vol. 19, no. 3, pp. 177-185, 1992.

[3] Q. Hu, G. Ma, and L. Xie, "Robust and adaptive variable structure output feedback control of uncertain systems with input nonlinearity," Automatica, vol. 44, no. 2, pp. 552-559, 2008.

[4] H. Zhang, X. Zhang, and J. Wang, "Robust gain-scheduling energy-to-peak control of vehicle lateral dynamics stabilisation," Vehicle System Dynamics, vol. 52, no. 3, pp. 309-340, 2014.

[5] Y. Shi, H. Zhang, and J. Wang, "On energy-to-peak filtering for nonuniformly sampled nonlinear systems: a Markovian jump system approach," IEEE Transactions on Fuzzy Systems, vol. 22, no. 1, pp. 212-222, 2014.

[6] H. Zhang, Y. Shi, and J. Wang, "Observer-based tracking controller design for networked predictive control systems with uncertain Markov delays," International Journal of Control, vol. 86, no. 10, pp. 1824-1836, 2013.

[7] H. Zhang, Y. Shi, and M. Liu, " $\mathrm{H}_{\infty}$ step tracking control for networked discrete-time nonlinear systems with integral and predictive actions," IEEE Transactions on Industrial Informatics, vol. 9, no. 1, pp. 337-345, 2013.

[8] H. Zhang and Y. Shi, "Observer-based $H^{\infty}$ feedback control for arbitrarily time-varying discrete-time systems with intermittent measurements and input constraints," Journal of Dynamic Systems, Measurement and Control, vol. 134, no. 6, Article ID 061008, 2012.

[9] M. Chadli and H. R. Karimi, "Robust observer design for unknown inputs takagi-sugeno models," IEEE Transactions on Fuzzy Systems, vol. 21, no. 1, pp. 158-164, 2013.

[10] M. Zapateiro, N. Luo, H. R. Karimi, and J. Vehí, "Vibration control of a class of semiactive suspension system using neural network and backstepping techniques," Mechanical Systems and Signal Processing, vol. 23, no. 6, pp. 1946-1953, 2009.

[11] M. Zapateiro, H. R. Karimi, and N. Luo, "Semiactive vibration control of nonlinear structures through adaptive backstepping techniques with $H_{\infty}$ performance," International Journal of Systems Science, vol. 42, no. 5, pp. 853-861, 2011.

[12] S. Yin, S. X. Ding, A. Haghani, H. Hao, and P. Zhang, "A comparison study of basic data-driven fault diagnosis and process monitoring methods on the benchmark Tennessee Eastman process," Journal of Process Control, vol. 22, no. 9, pp. 1567-1581, 2012. 
[13] S. Yin, G. Wang, and H. R. Karimi, "Data-driven design of robust fault detection system for wind turbines," Mechatronics, vol. 24, no. 4, pp. 298-306, 2013.

[14] S. Yin, X. Yang, and H. R. Karimi, "Data-driven adaptive observer for fault diagnosis," Mathematical Problems in Engineering, vol. 2012, Article ID 832836, 21 pages, 2012.

[15] S. Aouaouda, M. Chadli, P. Shi, and H. R. Karimi, "Discretetime $H_{-} / H_{\infty}$ sensor fault detection observer design for nonlinear systems with parameter uncertainty," International Journal of Robust and Nonlinear Control, 2013.

[16] S. Yin, S. Ding, and H. Luo, "Real-time implementation of fault tolerant control system with performance optimization," IEEE Transactions on Industrial Electronics, vol. 61, no. 5, pp. 24022411, 2014.

[17] H. Li, H. Gao, P. Shi, and X. Zhao, "Fault-tolerant control of Markovian jump stochastic system with augmented sliding mode observer approach," Automatica. In press.

[18] H. Li, H. Liu, H. Gao, and P. Shi, "Reliable fuzzy control for active suspension systems with actuator delay and fault," IEEE Transactions on Fuzzy Systems, vol. 20, no. 2, pp. 342-357, 2012.

[19] X. Su, P. Shi, L. Wu, and Y. Song, "A novel control design on discrete-time takagi-sugeno fuzzy systems with time-varying delays," IEEE Transactions on Fuzzy Systems, vol. 21, no. 4, pp. 655-671, 2013.

[20] S. Yin, S. X. Ding, A. H. A. Sari, and H. Hao, "Data-driven monitoring for stochastic systems and its application on batch process," International Journal of Systems Science, vol. 44, no. 7, pp. 1366-1376, 2013.

[21] X. D. Zhao, H. Liu, and J. F. Zhang, "Multiple-mode observer design for a class of switched linear systems linear systems," IEEE Transactions on Automation Science and Engineering, 2013.

[22] X. D. Zhao, L. X. Zhang, P. Shi, and H. R. Karimi, "Robust control of continuous-time systems with state-dependent uncertainties and its application to Electronic Circuits," IEEE Transactions on Industrial Electronics, vol. 61, no. 8, pp. 4161-4170, 2014.

[23] M. Chadli, H. R. Karimi, and P. Shi, "On stability and stabilization of singular uncertain Takagi-Sugeno fuzzy systems," Journal of the Franklin Institute: Engineering and Applied Mathematics, vol. 351, no. 3, pp. 1453-1463, 2014.

[24] H. Wang, B. Chen, and C. Lin, "Adaptive fuzzy control for purefeedback stochastic nonlinear systems with unknown deadzone input," International Journal of Systems Science, vol. 2013, Article ID 773470, 2013.

[25] M. Chadli, A. Abdo, and S. X. Ding, " $H_{-} / H_{\infty}$ fault detection filter design for discrete-time Takagi-Sugeno fuzzy system," Automatica, vol. 49, no. 7, pp. 1996-2005, 2013.

[26] X. J. Su, P. Shi, L. G. Wu, and S. K. Nguang, "Induced $l_{2}$ filtering of fuzzy stochastic systems with time-varying delays," IEEE Transactions on Cybernetics, vol. 43, no. 4, pp. 1251-1264, 2013.

[27] H. Y. Li, X. J. Jing, H. K. Lam, and P. Shi, "Fuzzy sampled-data control for uncertain vehicle suspension systems," IEEE Transactions on Cybernetics, vol. 44, no. 7, pp. 1111-1126, 2013.

[28] H. Gao, Y. Zhao, and T. Chen, "Ho fuzzy control of nonlinear systems under unreliable communication links," IEEE Transactions on Fuzzy Systems, vol. 17, no. 2, pp. 265-278, 2009.

[29] H. J. Gao, X. M. Liu, and J. Lam, "Stability analysis and stabilization for discrete-time fuzzy systems with time-varying delay," IEEE Transactions on Systems, Man, and Cybernetics B: Cybernetics, vol. 39, no. 2, pp. 306-317, 2009.

[30] M. Chen, S. S. Ge, B. V. E. How, and Y. S. Choo, "Robust adaptive position mooring control for marine vessels," IEEE Transactions on Control Systems Technology, vol. 21, no. 2, pp. 395-409, 2013.
[31] M. Chen, S. S. Ge, and B. Ren, "Adaptive tracking control of uncertain MIMO nonlinear systems with input constraints," Automatica, vol. 47, no. 3, pp. 452-465, 2011.

[32] H. R. Karimi and H. Gao, "New delay-dependent exponential $H \infty$ synchronization for uncertain neural networks with mixed time delays," IEEE Transactions on Systems, Man, and Cybernetics B: Cybernetics, vol. 40, no. 1, pp. 173-185, 2010.

[33] S. Sefriti, J. Boumhidi, M. Benyakhlef, and I. Boumhidi, "Adaptive decentralized sliding mode neural network control of a class of nonlinear interconnected systems," International Journal of Innovative Computing, Information and Control, vol. 9, no. 7, pp. 2941-2947, 2013.

[34] H. Q. Wang, B. Chen, X. P. Liu, K. F. Liu, and C. Lin, "Robust adaptive fuzzy tracking control for pure-feedback stochastic nonlinear systems with input constraints," IEEE Transactions on Cybernetics, vol. 43, no. 6, pp. 2093-2104, 2013.

[35] L. Liu and X. J. Xie, "Output-feedback stabilization for stochastic high-order nonlinear systems with time-varying delay," Automatica, vol. 47, no. 12, pp. 2772-2779, 2011.

[36] S. S. Ge and C. Wang, "Direct adaptive NN control of a class of nonlinear systems," IEEE Transactions on Neural Networks, vol. 13, no. 1, pp. 214-221, 2002.

[37] S. S. Ge and K. P. Tee, "Approximation-based control of nonlinear MIMO time-delay systems," Automatica, vol. 43, no. 1, pp. 31-43, 2007.

[38] C. Wang, M. Wang, T. Liu, and D. J. Hill, "Learning from ISS-modular adaptive NN control of nonlinear strict-feedback systems," IEEE Transactions on Neural Networks and Learning Systems, vol. 23, no. 10, pp. 1539-1550, 2012.

[39] H. Q. Wang, B. Chen, and C. Lin, "Adaptive neural tracking control for a class of stochastic nonlinear systems with unknown dead-zone," International Journal of Innovative Computing, Information and Control, vol. 9, no. 8, pp. 3257-3270, 2013.

[40] H. Q. Wang, B. Chen, K. F. Liu, X. P. Liu, and C. Lin, "Adaptive neural tracking control for a class of nonstrict-feedback stochastic nonlinear systems with unknown backlashlike hysteresis," IEEE Transactions on Neural Networks and Learning Systems, vol. 25, no. 5, pp. 947-958, 2014.

[41] T. Zhang and S. S. Ge, "Adaptive neural network tracking control of MIMO nonlinear systems with unknown dead zones and control directions," IEEE Transactions on Neural Networks, vol. 20, no. 3, pp. 483-497, 2009.

[42] T. S. Li, D. Wang, G. Feng, and S. C. Tong, "A DSC approach to robust adaptive $\mathrm{NN}$ tracking control for strict-feedback nonlinear systems," IEEE Transactions on Systems, Man, and Cybernetics B: Cybernetics, vol. 40, no. 3, pp. 915-927, 2010.

[43] Y. Liu, C. L. P. Chen, G. Wen, and S. Tong, "Adaptive neural output feedback tracking control for a class of uncertain discrete-time nonlinear systems," IEEE Transactions on Neural Networks, vol. 22, no. 7, pp. 1162-1167, 2011.

[44] X. D. Zhao, L. X. Zhang, P. Shi, and H. R. Karimi, "Novel stability criteria for T-S fuzzy systems," IEEE Transactions on Fuzzy Systems, vol. 22, no. 2, pp. 313-323, 2014.

[45] Y. Yang, G. Feng, and J. Ren, "A combined backstepping and small-gain approach to robust adaptive fuzzy control for strictfeedback nonlinear systems," IEEE Transactions on Systems, Man, and Cybernetics A: Systems and Humans., vol. 34, no. 3, pp. 406-420, 2004.

[46] S. Tong, Y. Li, and P. Shi, "Observer-based adaptive fuzzy backstepping output feedback control of uncertain MIMO purefeedback nonlinear systems," IEEE Transactions on Fuzzy Systems, vol. 20, no. 4, pp. 771-785, 2012. 
[47] S. Tong and Y. Li, "Observer-based fuzzy adaptive control for strict-feedback nonlinear systems," Fuzzy Sets and Systems, vol. 160, no. 12, pp. 1749-1764, 2009.

[48] S. C. Tong, Y. Li, Y. M. Li, and Y. J. Liu, "Observer-based adaptive fuzzy backstepping control for a class of stochastic nonlinear strict-feedback systems," IEEE Transactions on Systems, Man and Cybernetics, Part B: Cybernetics, vol. 41, no. 6, pp. 1693-1704, 2011.

[49] S. C. Tong, T. Wang, Y. M. Li, and B. Chen, "A combined backstepping and stochastic small-gain approach to robust adaptive fuzzy output feedback control," IEEE Transactions on Fuzzy Systems, vol. 21, no. 2, pp. 314-327, 2013.

[50] B. Chen, X. Liu, K. Liu, and C. Lin, "Direct adaptive fuzzy control of nonlinear strict-feedback systems," Automatica, vol. 45, no. 6, pp. 1530-1535, 2009.

[51] S. Bououden, M. Chadli, F. Allouani, and S. Filali, "A new approach for fuzzy predictive adaptive controller design using particle swarm optimization algorithm," International Journal of Innovative Computing, Information and Control, vol. 9, no. 9, pp. 3741-3758, 2013.

[52] B. Chen, X. Liu, K. Liu, and C. Lin, "Fuzzy-approximationbased adaptive control of strict-feedback nonlinear systems with time delays," IEEE Transactions on Fuzzy Systems, vol. 18, no. 5, pp. 883-892, 2010.

[53] M. Wang, B. Chen, X. Liu, and P. Shi, "Adaptive fuzzy tracking control for a class of perturbed strict-feedback nonlinear timedelay systems," Fuzzy Sets and Systems, vol. 159, no. 8, pp. 949967, 2008.

[54] Y. Li, S. Tong, and T. Li, "Adaptive fuzzy output feedback control of uncertain nonlinear systems with unknown backlash-like hysteresis," Information Sciences, vol. 198, pp. 130-146, 2012.

[55] Q. Zhou, P. Shi, J. Lu, and S. Xu, "Adaptive output-feedback fuzzy tracking control for a class of nonlinear systems," IEEE Transactions on Fuzzy Systems, vol. 19, no. 5, pp. 972-982, 2011.

[56] Z. P. Jiang and L. Praly, "Design of robust adaptive controllers for nonlinear systems with dynamic uncertainties," Automatica, vol. 34, no. 7, pp. 825-840, 1998.

[57] Z. J. Wu, X. J. Xie, and P. Shi, "Robust adaptive output-feedback control for nonlinear systems with output unmodeled dynamics," International Journal of Robust and Nonlinear Control, vol. 18, no. 11, pp. 1162-1187, 2008.

[58] S. Tong, Y. Li, and P. Shi, "Fuzzy adaptive backstepping robust control for SISO nonlinear system with dynamic uncertainties," Information Sciences, vol. 179, no. 9, pp. 1319-1332, 2009.

[59] S. C. Tong and Y. M. Li, "Fuzzy adaptive robust backstepping stabilization for SISO nonlinear systems with unknown virtual control direction," Information Sciences, vol. 180, no. 23, pp. 4619-4640, 2010.

[60] R. M. Sanner and J. E. Slotine, "Gaussian networks for direct adaptive control," IEEE Transactions on Neural Networks, vol. 3, no. 6, pp. 837-863, 1992. 


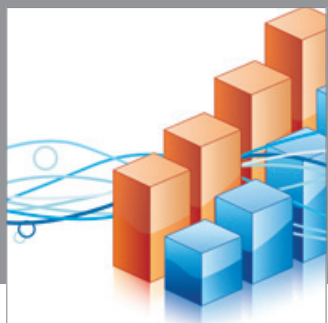

Advances in

Operations Research

mansans

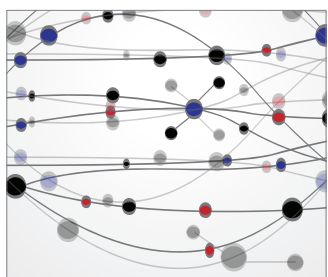

The Scientific World Journal
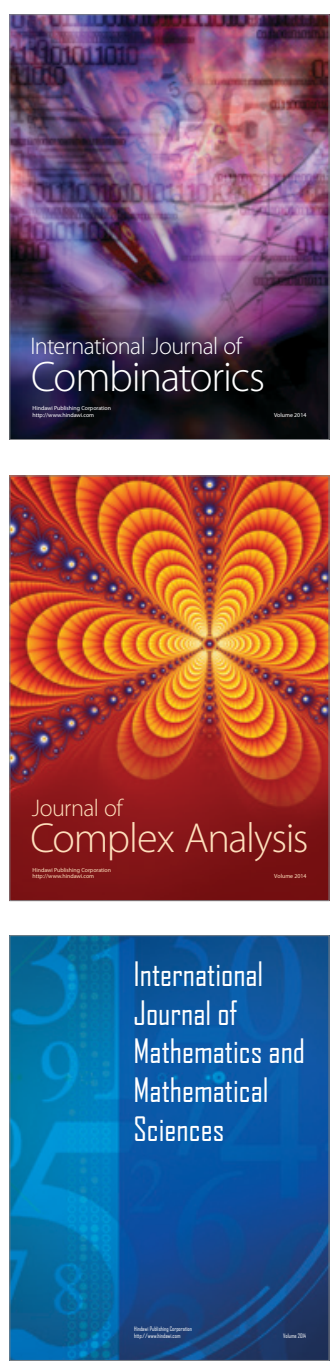
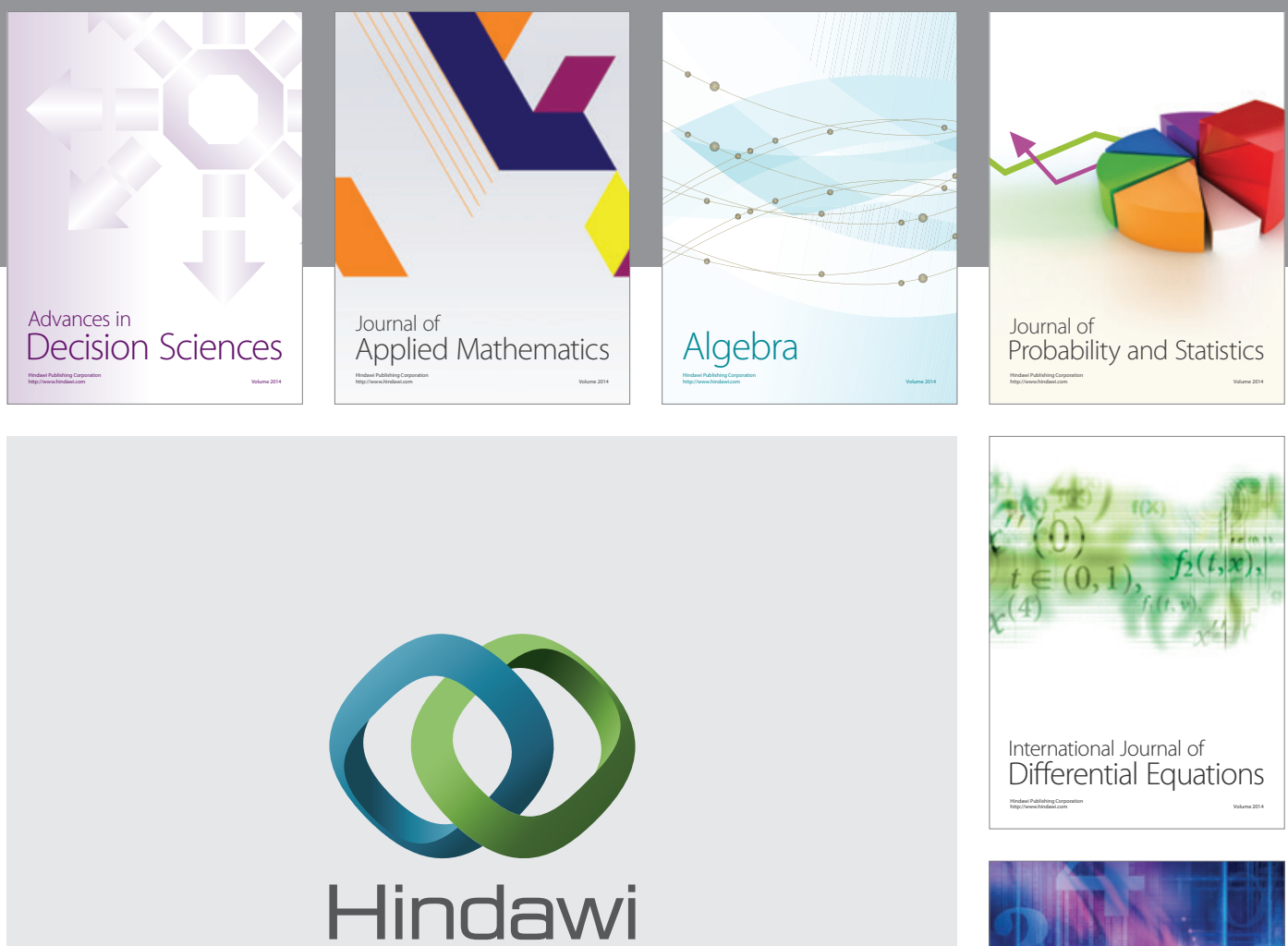

Submit your manuscripts at http://www.hindawi.com
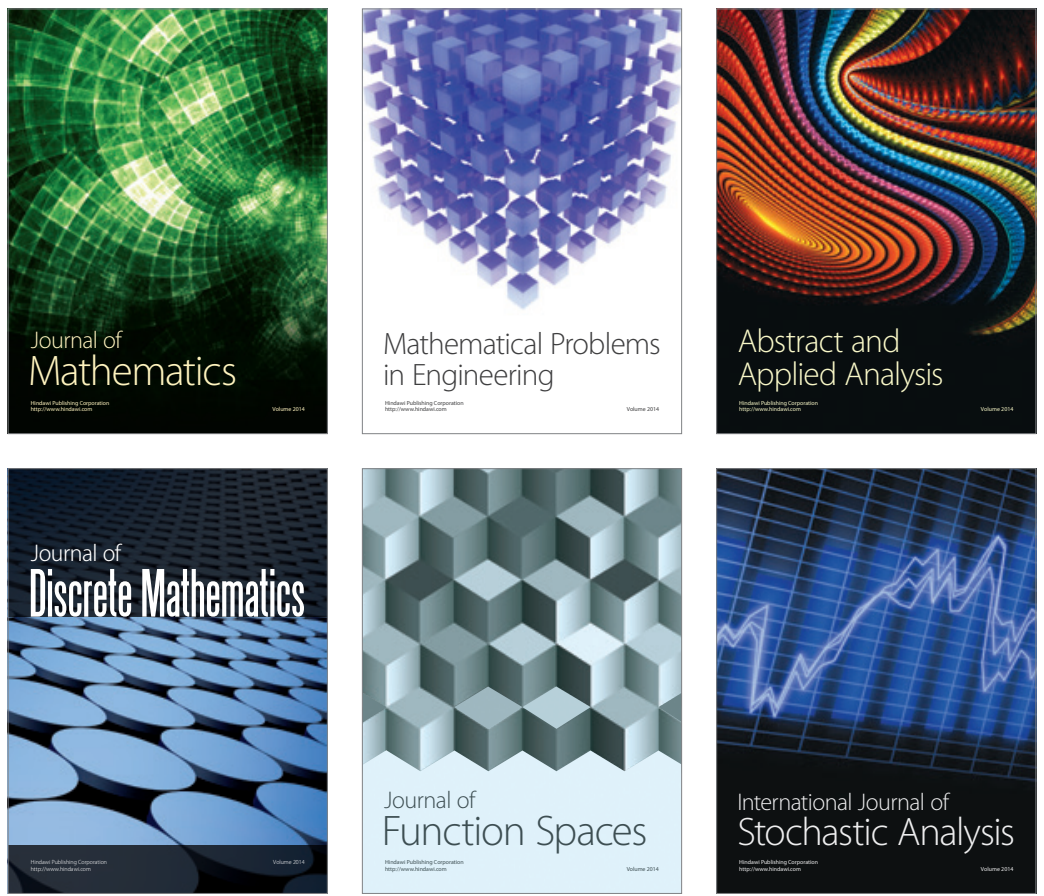

Journal of

Function Spaces

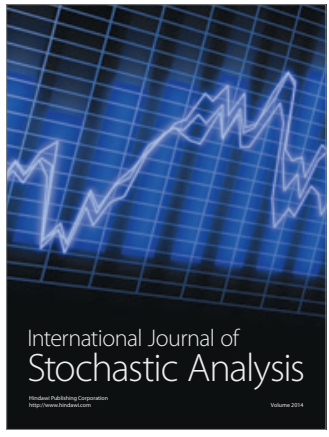

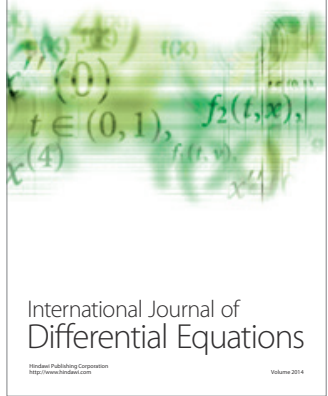
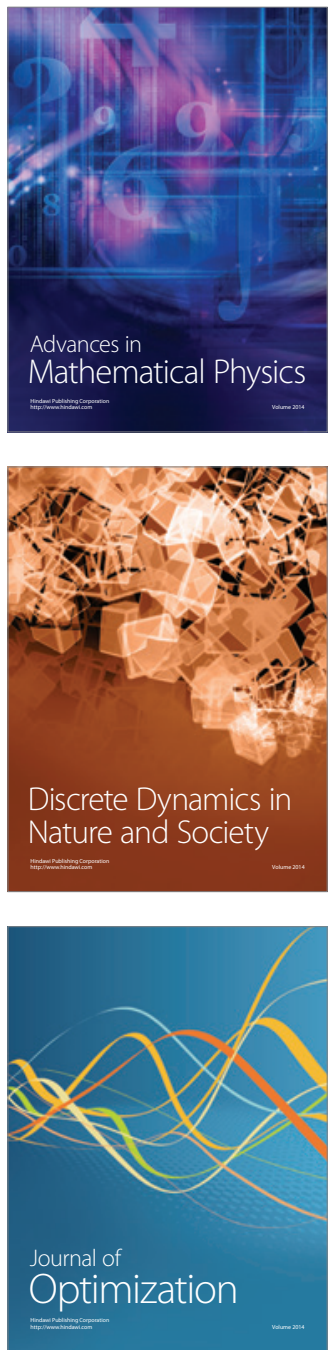